\title{
Synthesis and Cytotoxicity of Heterocyclic Compounds Derived from Cyclohexane-1,3-Dione
}

\author{
Mohamed A. Shaaban, Mona M. Kamel*, Yara R. Milad \\ Department of Pharmaceutical Organic Chemistry, Faculty of Pharmacy, Cairo University, Giza, Egypt \\ Email: monakamel116@yahoo.com
}

Received 25 September 2014; revised 29 October 2014; accepted 1 December 2014

Copyright (C) 2014 by authors and OALib.

This work is licensed under the Creative Commons Attribution International License (CC BY). http://creativecommons.org/licenses/by/4.0/

(c) (i) Open Access

\begin{abstract}
Cyclohexane-1,3-dione (1) was used as a template to develop new anticancer compounds. The ring modification of compound 1occurred through its reaction with either aromaticaldehydes or benzenediazonium chloride to produce the corresponding products. The latter compounds underwenttheterocyclization reactions through the reaction with elemental sulfur and some active methylene reagents to produce tetrahydrobenzo[b]thiophene derivatives. The reaction of compound 1 with elemental sulfur and phenylisothiocyanategave the tetrahydrobenzo[ $d]$ thiazole derivative. The cytotoxicity of the newly synthesized products against human cancer and normal cell lines was evaluated. Some compounds showed high cytotoxicity against cancer cell lines. The results showed that compounds 3b, 5c, 7b, 10b, 12, 14b, 16, 18b, 19b, 20b, 21 and 24 showed the highest cytotoxicity. Moreover, the toxicity of twelve active compounds were measured.
\end{abstract}

\section{Keywords}

Cyclohexane-1,3-Dione, Thiophene, Thiazole, Pyridazine, Cytotoxicity

Subject Areas: Biochemistry, Biotechnology

\section{Introduction}

As typical reactive 1,3-dicarbonyl compounds, cyclohexane-1,3-dione and its analogy 5,5-dimethyl cyclohexane-1,3-dione(dimedone) have been widely used in versatile synthetic reactions [1]-[5]. Cyclohexan-1,3-dione is not only a typical reagent for Knoevenagel condensation, but also adds easily to electron-deficient alkenes via Michael addition. On the other hand, its one or two carbonyl groups could take part in substitution and cyclization reactions through the tautomerizedenolate form. Thus, the cascade reactions of addition, elimination and

${ }^{*}$ Corresponding author.

How to cite this paper: Shaaban, M.A., Kamel, M.M. and Milad, Y.R. (2014) Synthesis and Cytotoxicity of Heterocyclic Compounds Derived from Cyclohexane-1,3-Dione. Open Access Library Journal, 1: e1115.

http://dx.doi.org/10.4236/oalib.1101115 
substitution could be achieved in many reactions involving cyclohexan-1,3-dione moiety. On the other hand, 1,3-dicarbonyl derivatives constitute important synthetic intermediates, incorporating multiple functionalities that can be involved either as nucleophilic or electrophilic species in a large variety of synthetic transformations [6]-[8]. Moreover, cyclohexane-1,3-dione derivatives play an important role in organic synthesis due to their usefulness in the preparation of many biologically important compounds [9]. Many herbicides having cyclohexane-1,3-dione backbone such as tralkoxydim, sethoxydim orclethodim are well known [10] [11]. In previous work we were interested in the design, screening, synthesis and biological evaluation of tetrahydrobenzo[b]thiophene as anti-cancer agents [12]. In addition, it has been reported that some heterocyclic derivatives with different substituents showed high potency [13] [14]. In an attempt to obtain an antitumor agent with high activity, the substitution pattern at positions 1 and 2 of the cyclohexan-1,3-dione to produce 2-cyclohexene-1-one pharmacophore [15] [16] was selected in order to alter the electronic environment and thus affect the lipophilicity of the target molecules. Thus, in the present work we are demonstrating the uses of cyclohexan-1,3-dione to synthesis fused thiophene, pyran and pyridazine derivatives incorporated 2-cyclohexene-1-one moiety with varieties of functional groups followed by the evaluation of the newly synthesized products towards human cancer and normal cell lines.

\section{Results and Discussion}

Herein, in order to extend our research on anticancer heterocyclic derivatives with high inhibitory effects toward some cancer cell lines, we report the synthesis of new fused thiophene, thiazole, pyran, pyridazineand 1,2,4-triazine derivatives derived from cyclohexane-1,3-dione (1). Moreover, some the newly synthesized products were good candidates as anticancer drugs through their screening towards cancer and normal cell lines. Thus, the reaction of compound $\mathbf{1}$ with either benzaldehyde or 4-methoxybenzaldehyde gave the corresponding arylidene derivatives 3a, b. Structures of the latter products were established based on analytical and spectral data. Compounds $\mathbf{3 a}$ and $\mathbf{3 b}$ were good candidates for many heterocyclization reactions. Thus, the reaction of eithercompounds $\mathbf{3 a}$ and $\mathbf{3 b}$ with elemental sulfur and either malononitrile or ethyl cyanoacetate gave the tetrahydrobenzo [b]thiophene derivatives 5a-d, respectively. Formation of the latter products was explained in terms of the well known Gewald's thiophene synthesis [17]. The ${ }^{1} \mathrm{H}$ NMR and ${ }^{13} \mathrm{C}$ NMR were the basis for the structure elucidation of compounds 5a-d.

Similarly the reaction of either $\mathbf{3 a}$ or $\mathbf{3 b}$ with elemental sulfur and the 2-aminoprop-1-ene-1,1,3-tricarbonitrile (6) gave the tetrahydrobenzo[b]thiophene derivatives $7 \mathbf{a}$ and $\mathbf{7 b}$, respectively (Scheme 1 ). The analytical and spectral data of $\mathbf{7 a}$ and $\mathbf{7 b}$ were in agreement with their respective structures (see experimental section).

On the other hand, the reaction of cyclohexan-1,3-dione with elemental sulfur and the 2-aminoprop-1-ene-1, 1,3-tricarbonitrile(6) gave the tetrahydrobenzo-[4,5]thieno[2,3-b]pyridine derivative $\mathbf{9}$. Compound $\mathbf{9}$ was formed via the intermediate formation of $\mathbf{8}$ followed the Michael addition of the $\mathrm{NH}_{2}$ group to one of the two CN groups.

$4 H$-Benzo[b]pyran derivatives form an important class of heterocyclic compounds having remarkable pharmaceutical and biological activities. Therefore, the $4 H$-benzo[b]pyrans received significant amount of attention from pharmaceutical and organic chemistry communities. The commonly used method for the synthesis of $4 \mathrm{H}$ benzo[b]pyrans is the condensation of aldehyde, cinnamonitrile derivatives and carbonyl compounds in the presence of acidic or basic catalysts. With optimized reaction condition in hand, our trials to synthesis pyran derivatives using another reaction route starting from compounds 3a and either of malononitrile (4a) or ethyl cyanoacetate (4b) gave the tetrahydrobenzo[b]pyran derivatives 10a, 10b; respectively (Scheme 2 ). The analytical and spectral data of compounds 10a, 10b were in analogy with their respective structures. Further confirmations for structures of 10a, 10b were obtained through their synthesis via another reaction route. Thus, the reaction of cyclohexan-1,3-dione with either $\alpha$-cyanocinnamonitrile (11a) or ethyl $\alpha$-cyanocinnamate (11b) gave the same products 10a, 10b (same m.p., mixed m.p. and fingerprint IR).

Cyclohexan-1,3-dione (1) reacted with 2-aminoprop-1-ene-1,1,3-tricarbonitrile (6) in absolute ethanol solution containing triethylamineto give the tetrahydronaphalene-2-one derivative 12. Formation of the latter product is explained in terms of the first water elimination followed by cyclization. On the other hand, the reaction of either compound 3a or $\mathbf{3 b}$ with the 2-aminoprop-1-ene-1,1,3-tricarbonitrile (6) in ethanol solution containing triethylamine gave tetrahydronaphthalene derivatives $14 \mathbf{a}$ and $\mathbf{1 4} \mathbf{b}$, respectively. The most important feature to confirm the structure of 14a (as an example) through the ${ }^{1} \mathrm{H}$ NMR spectrum that showed, beside the cyclohex- 


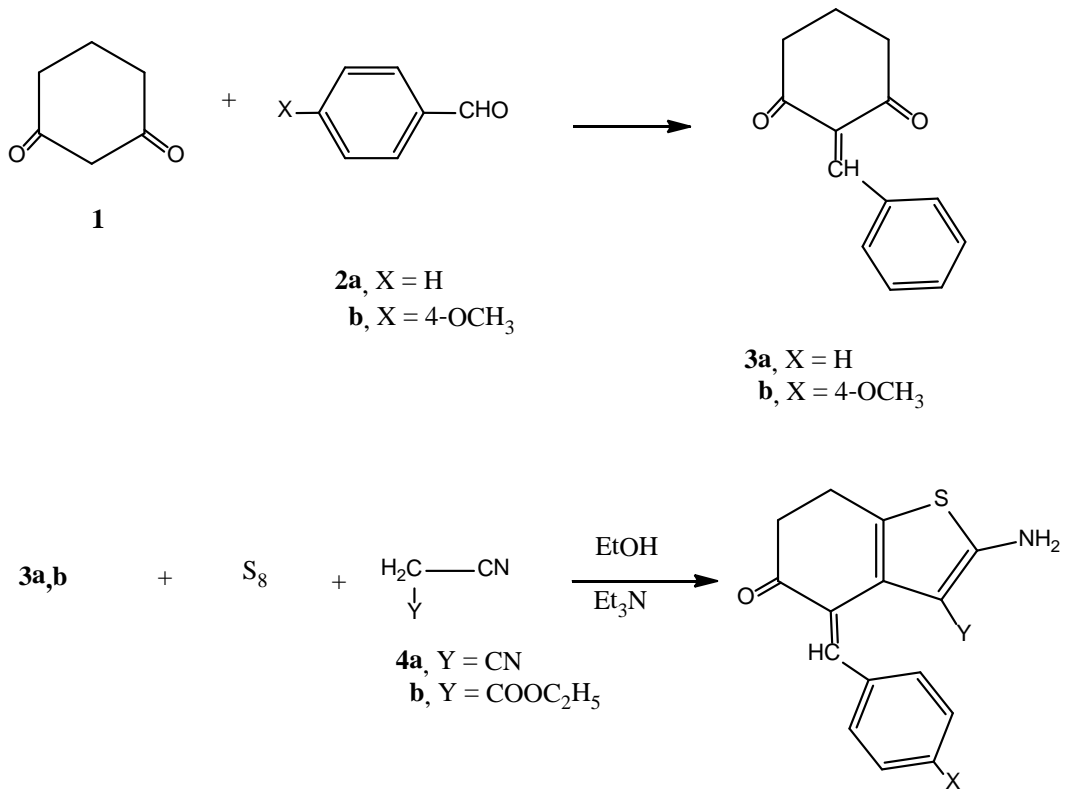

\begin{tabular}{l|l|l} 
5 & $\mathrm{X}$ & \multicolumn{1}{|}{$\mathrm{Y}$} \\
\hline $\mathrm{a}$ & $\mathrm{H}$ & $\mathrm{CN}$ \\
$\mathrm{b}$ & $\mathrm{H}$ & $\mathrm{COOEt}$ \\
$\mathbf{c}$ & $\mathrm{OCH}_{3}$ & $\mathrm{CN}$ \\
$\mathbf{d}$ & $\mathrm{OCH}_{3}$ & $\mathrm{COOEt}$
\end{tabular}

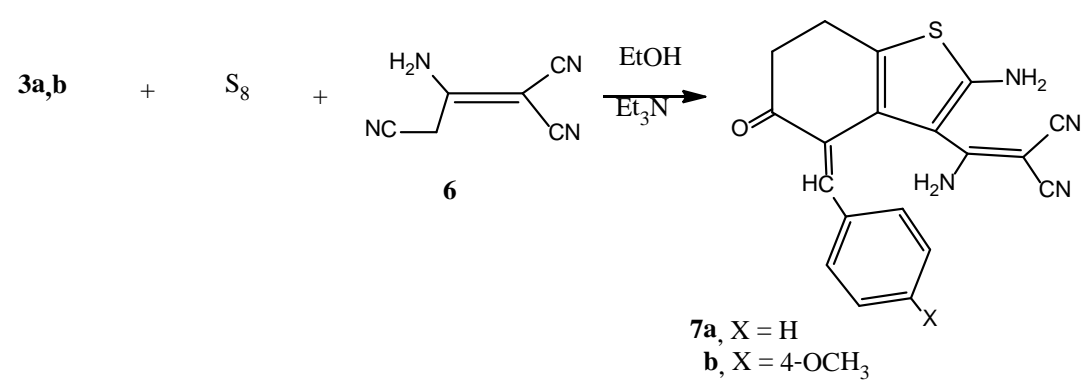

\section{Scheme 1. Synthesis of compounds 3a,b; 5a-d and 7a,b.}

ene and the phenyl moieties signals, two singlets $\left(\mathrm{D}_{2} \mathrm{O}\right.$ exchangeable) at $\delta 4.33,5.01 \mathrm{ppm}$ for the two $\mathrm{NH}_{2}$ groups and the ${ }^{13} \mathrm{C}$ NMR spectrum showed $\delta$ : 20.6, $36.8\left(2 \mathrm{CH}_{2}\right), 95.4,95.3(\mathrm{CH}=\mathrm{C}), 110.2(\mathrm{CH}=\underline{\mathrm{C}}), 116.8$, 117.3 (2 CN), 127.7, 128.8, 128.9, 129.0, 129.2, 129.8, 140.7 (two benzene C), 196.4 (C=O).

The reaction of the cyclohexane-1,3-dione with elemental sulfur and phenylisothiocyanate (15) in 1,4-dioxane solution containing triethylamine give the 3-phenyl-2-thioxo-2,3,5,6-tetrahydrobenzo[d]thiazol-7(4H)-one (16) (Scheme 3). The structure of the latter product was confirmed on the basis of ${ }^{1} \mathrm{H}$ NMR and ${ }^{13} \mathrm{C}$ NMR (see experimental section).

Next we moved through studying the reactivity of the cyclohexan-1,3-dione (1) towards arylhydrazone derivatives followed heterocyclization of the reaction products in the aim of producing new anticancer agents. Thus, the reaction of compound $\mathbf{1}$ with either benzenediazonium chloride (17a) or 4-chlorobenzenediazonium chloride (17b) gave the arylhydrazone derivatives 18a and 18b, respectively. The high yield of compound 18a encouraged us to use it for further chemical reactions. In fact compound 18b was synthesized in order to study the effect of the 4-chloro group through its cytotoxicity compared with 18a. The reaction of compound 18a with either malononitrile (4a) or ethyl cyanoacetate (4b) in the presence of ammonium acetate in an oil bath $120^{\circ} \mathrm{C}$ gave the 


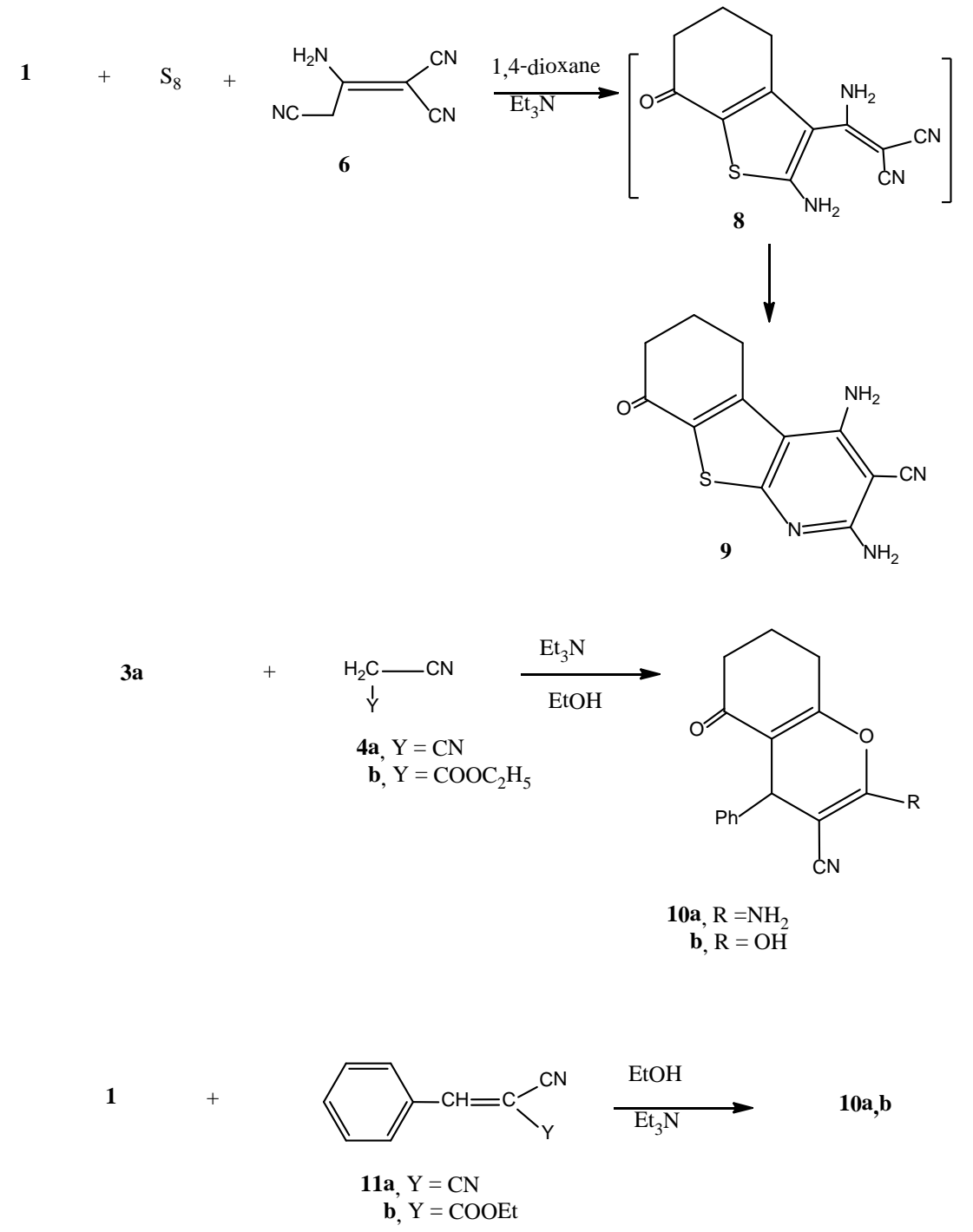

\section{Scheme 2. Synthesis of compounds $\mathbf{9}, \mathbf{1 0 b}$.}

Knoevenegal condensation products 19a, 19b, respectively. On the other hand, carrying the same reaction, but using 1,4-dioxane and triethylamine gave the 2,3,5,6,7,8-hexahydrocinnoline derivatives 20a, 20b; respectively (Scheme 4). Formation of the latter products were explained in terms of the intermediate formation of 19a, 19b followed by cyclization.

The reactivity of 18a towards thiophene synthesis applying the method of Gewald's thiophene was studied. Thus, the reaction of compound 18a with malononitrile (4a) and elemental sulfur in 1,4-dioxane containing a catalytic amount of triethylamine gave the cyclohexene[b]thiophenederivarive $\mathbf{2 1}$. On the other hand, the reaction of 18a with ethyl cyanoacetate (4b) and elemental sulfur gave the annulated derivative $\mathbf{2 2}$. The structure of the latter product was assigned based on the elemental analysis and other spectroscopic data (see experimental section).

The reaction of compound 18a with phenylisothiocyanate $(\mathbf{1 3})$ gave cyclohexene[e]triazine derivative $\mathbf{2 3}$. Finally we studied the reactivity of compound 18a with 2-aminoprop-1-ene-1,1,3-tricarbonitrile (6) using different reaction conditions. Thus, the reaction of 18a with compound $\mathbf{6}$ in the presence of ammonium acetate at $120^{\circ} \mathrm{C}$ gave the cyclohexene[ $b$ ]pyridazine 24 derivative. On the other hand, carrying the same reaction but in 1,4-dioxane containing a catalytic amount of triethylamine gave the 5,6,7,8-tetrahydronaphthalene derivative 25 (Scheme 5). 
<smiles>CCN(C)CCO</smiles>

12

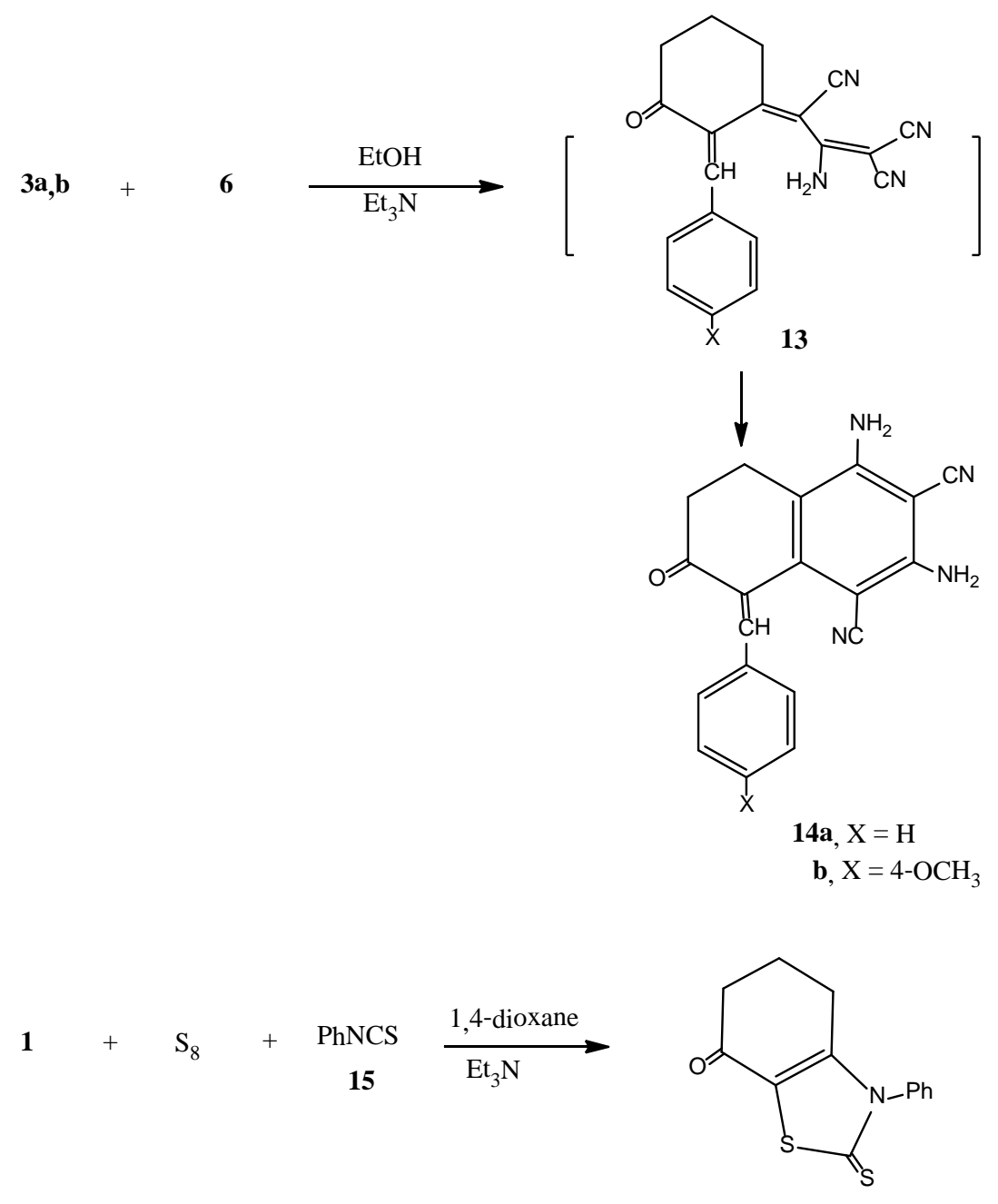

16

Scheme 3. Synthesis of compounds 12, 14a,b and 16.

\section{In Vitro Cytotoxic Assay}

\subsection{Chemicals}

Fetal bovine serum (FBS) and L-glutamine, were purchased from Gibco Invitrogen Co. (Scotland, UK). RPMI1640 medium was purchased from Cambrex (New Jersey, USA). Dimethyl sulfoxide (DMSO), doxorubicin, penicillin, streptomycin and sulforhodamine B (SRB) were purchased from Sigma Chemical Co. (Saint Louis, USA). 

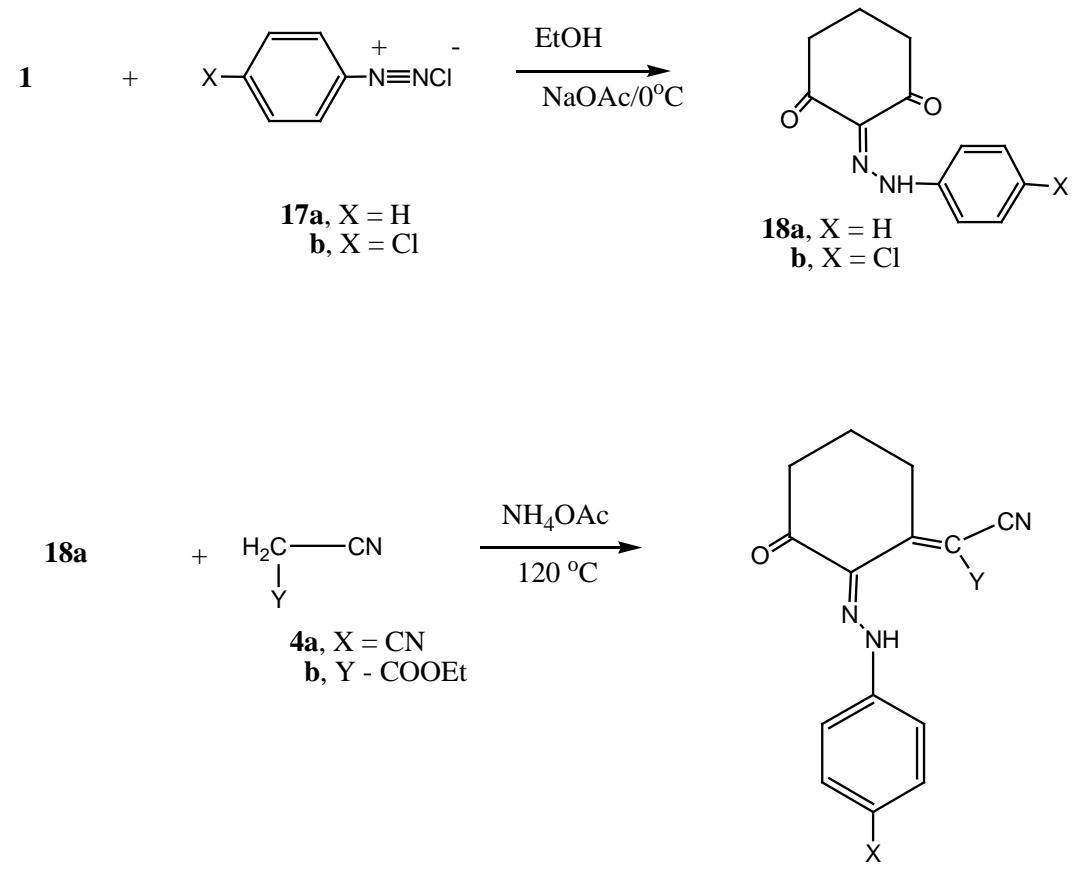

19a, $\mathrm{X}=\mathrm{H}, \mathrm{Y}=\mathrm{CN}$

b, $\mathrm{X}=\mathrm{H}, \mathrm{Y}=\mathrm{COOEt}$

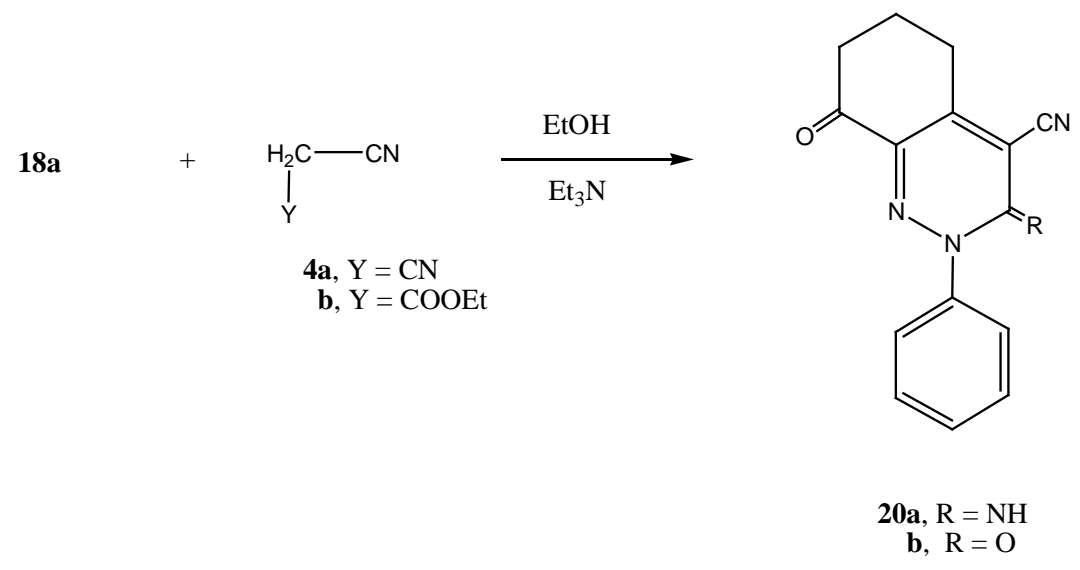

Scheme 4. Synthesis of compounds 18a,b; 19a,b and 20a,b.

\subsection{Cell Cultures}

The cell cultures were obtained from the European collection of cell cultures (ECACC, Salisbury, UK) and human gastric cancer (NUGC and HR), human colon cancer (DLD1), human liver cancer (HA22T and HEPG2), human breast cancer (MCF), nasopharyngeal carcinoma (HONE1) and normal fibroblast cells (WI38) were kindly provided by the National Cancer Institute (NCI, Cairo, Egypt). They grow as monolayer and routinely maintained in RPMI-1640 medium supplemented with 5\% heat inactivated FBS, 2 mM glutamine and antibiotics (penicillin $100 \mathrm{U} / \mathrm{mL}$, streptomycin $100 \mathrm{lg} / \mathrm{mL}$ ), at $37^{\circ} \mathrm{C}$ in a humidified atmosphere containing $5 \% \mathrm{CO}_{2}$. Exponentially growing cells were obtained by plating $1.5 \times 10^{5}$ cells $/ \mathrm{mL}$ for the seven human cancer cell lines including cells derived from $0.75 \times 10^{4}$ cells/mL followed by $24 \mathrm{~h}$ of incubation. The effect of the vehicle solvent (DMSO) on the growth of these cell lines was evaluated in all the experiments by exposing untreated control cells to the maximum concentration ( $0.5 \%$ ) of DMSO used in each assay. 


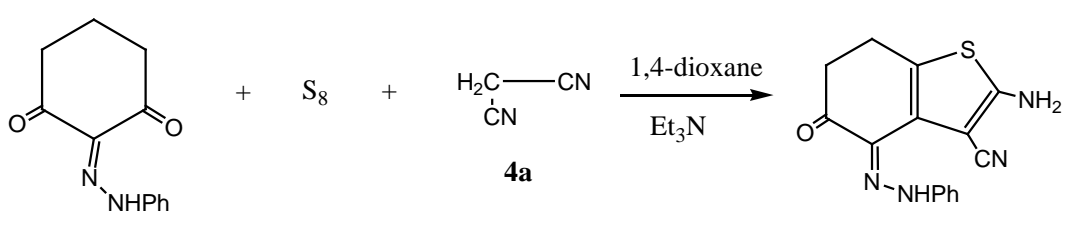

18a

21

$$
\text { 18a }+\mathrm{S}_{8}+\underset{\mathrm{CaO}}{\mathrm{C}}
$$

\section{Scheme 5. Synthesis of compounds 21-25.}

The heterocyclic compounds, prepared in this study, were evaluated according to standard protocols for their in vitro cytotoxicity against six human cancer cell lines including cells derived from human gastric cancer (NUGC), human colon cancer (DLD1), human liver cancer (HA22T and HEPG2), human breast cancer (MCF), nasopharyngeal carcinoma (HONE1) and a normal fibroblast cells (WI38). All of IC 50 values were listed in Table 1. Some heterocyclic compounds was observed with significant cytotoxicity against most of the cancer cell lines tested $\left(\mathrm{IC}_{50}=10-1000 \mathrm{nM}\right)$. Normal fibroblasts cells (WI38) were affected to a much lesser extent $\left(\mathrm{IC}_{50}>\right.$ $10,000 \mathrm{nM}$ ). The reference compound used is the CHS-828 which is a pyridylcyanoguanidine antitumor agent.

\subsection{Structure Activity Relationship}

From Table 1 it is clear that the cyclohexene moiety was found to be crucial for the cytotoxic effect of the cyclic compounds 3a, 3b-25. Compounds 3b, 5c, 7b, 10b, 12, 14b, 16, 18b, 19b, 20b, 21 and 24 exhibited optimal 
Table 1. Cytotoxicity of the newly synthesized products against a variety of cancer cell lines $\left[\mathrm{IC}_{50}^{\mathrm{b}}(\mathrm{nM})\right]$.

\begin{tabular}{|c|c|c|c|c|c|c|c|}
\hline \multicolumn{8}{|c|}{ Compd Cytotoxocity ( $\mathrm{IC}_{50}$ in nM) } \\
\hline & NUGC & DLDI & HA22T & HEPG2 & HONE1 & MCF & WI38 \\
\hline 3a & 3342 & 4220 & 3310 & 1328 & 2824 & 3243 & 380 \\
\hline $3 \mathbf{b}$ & 84 & 46 & 233 & 250 & 220 & 338 & 422 \\
\hline $5 a$ & 2101 & 1380 & 2258 & 2166 & 2180 & 2330 & 512 \\
\hline $5 \mathbf{b}$ & 1135 & 1240 & 1278 & 2359 & 1266 & 2555 & 128 \\
\hline $5 c$ & 38 & 46 & 120 & 337 & 441 & 180 & 320 \\
\hline 5d & 1222 & 3390 & 2063 & 2440 & 2177 & 3230 & 882 \\
\hline $7 a$ & 2228 & 2342 & 2100 & 655 & 2528 & 2260 & 640 \\
\hline $7 \mathbf{b}$ & 122 & 30 & 59 & 270 & 1140 & 1160 & 260 \\
\hline 9 & 2322 & 1159 & 2253 & 3370 & 2326 & 2270 & 380 \\
\hline $10 a$ & 2265 & 1220 & 2257 & 3228 & 2250 & 2220 & 466 \\
\hline $10 \mathrm{~b}$ & 338 & 232 & 228 & 28 & 84 & 2224 & 589 \\
\hline 12 & 1180 & 3268 & 2560 & 2128 & 3330 & 1180 & 280 \\
\hline $14 a$ & 1235 & 3160 & 2061 & 3218 & 1186 & 1693 & 380 \\
\hline $14 b$ & 2120 & 2240 & 1120 & 2130 & 2348 & 2254 & 120 \\
\hline 16 & 22 & 38 & 48 & 20 & 33 & 73 & 320 \\
\hline $18 a$ & 3270 & 1690 & 1155 & 2320 & 440 & 2657 & 540 \\
\hline $18 \mathrm{~b}$ & 1355 & 160 & 290 & 221 & 2229 & 2332 & 631 \\
\hline 19a & 2280 & 2455 & 1884 & 2562 & 2310 & 3148 & 128 \\
\hline $19 b$ & 1480 & 1150 & 1140 & 1328 & 1260 & 1140 & 220 \\
\hline $20 a$ & 1148 & 2163 & 3063 & 2232 & 1480 & 3860 & 890 \\
\hline $20 \mathrm{~b}$ & 620 & 255 & 760 & 520 & 2088 & 1264 & 634 \\
\hline 21 & 120 & 441 & 262 & 350 & 472 & 325 & 552 \\
\hline 22 & 1145 & 3210 & 3218 & 2276 & 2672 & 2711 & 493 \\
\hline 23 & 3320 & 2366 & 2781 & 3744 & 1589 & 1130 & 650 \\
\hline 24 & 38 & 46 & 122 & 320 & 480 & 226 & 380 \\
\hline 25 & 1153 & 2439 & 2670 & 1266 & 3200 & 3266 & 283 \\
\hline CHS 828 & 25 & 2315 & 2067 & 1245 & 15 & 18 & 378 \\
\hline
\end{tabular}

${ }^{a}$ NUGC, gastric cancer; DLDI, colon cancer; HA22T, liver cancer; HEPG2, liver cancer; HONEI, nasopharyngeal carcinoma; HR, gastric cancer; MCF, breast cancer; WI38, normal fibroblast cells. ${ }^{\text {b}}$ The sample concentration produces a $50 \%$ reduction in cell growth. CHS-828 is a pyridylcyanoguanidine anti-tumor agent. ${ }^{\mathrm{C}} \mathrm{IC}_{50}$ against the normal fibroblast cells (WI38) are indicated as multiples of $10^{4} \mathrm{nM}$.

cytotoxic effect against cancer cell lines, with $\mathrm{IC}_{50}$ 's in the $\mathrm{nM}$ range. Comparing the cytotoxicity of the benzylidenecyclohexane $\mathbf{3} \mathbf{a}$ and $\mathbf{3 b}$, it is obvious that the cytotoxicity of $\mathbf{3 b}$ is higher than that of $\mathbf{3 a}$. The presence of the $4-\mathrm{OCH}_{3}$ group in $\mathbf{3 b}$ is responsible for its high potency. Considering the 4,5,6,7-tetrahydrobenzo[b]thiophene derivatives $\mathbf{5 a - d}$, it is clear that the cytotoxicity of $\mathbf{5 c}$ is higher than those of $\mathbf{5 a}, \mathbf{5 b}$ and $\mathbf{5 d}$. Such high cytotoxicity of $\mathbf{5 c}$ is attributed to the presence of the $4-\mathrm{OCH}_{3}$ aryl moiety together with the 3-cyanothiophene moiety. The high cytotoxicity of compound $\mathbf{7 b}$ relative to compound $\mathbf{7 a}$ is also explained in terms of the presence of the 4- $\mathrm{OCH}_{3}$ aryl moiety. On the other hand, by considering the 5,6,7,8-tetrahydro- $4 \mathrm{H}$-chromene derivatives 10a, 10b it is clear that the presence of the 2-hydroxy group present in 10b is responsible for its high potency. The 5,6,7,8-tetrahydronaphthalene derivatives 12 and 14a, 14b showed low cytotoxicity effect towards the six cancer cell lines.

The 3-phenyl-2-thioxo-2,3,5,6-tetrahydrobenzo[d]thiazol-7(4H)-one(16) showed the maximum cytotoxicity among the tested compounds, this is attributed to the presence of the thiazole, thioxo moieties. Considering 
arylhydrazone derivatives $\mathbf{1 8 a}$ and $\mathbf{1 8 b}$, it is noticed that $\mathbf{1 8 a}$ showed high cytotoxicity against HONE1 with IC $_{50}$ $440 \mathrm{nM}$. On the other hand the 4-chlorophenyl derivative 18b showed high cytotoxicity against the three cancer cell lines DLDI, HA22T and HEPG2 with IG 50 's of 160, 290 and $212 \mathrm{nM}$, respectively. The high oxygen content present in compounds $19 \mathrm{~b}$ and $20 \mathrm{~b}$ is responsible for the high cytotoxicity of such compounds over the low oxygen content compounds 19a and 20a (Scheme 4). Finally considering the two isomeric compounds 24 and 25 it is clear that the hexahydrocinnnolin-4-yl $\mathbf{2 4}$ has higher cytotoxicity against the six cancer cell lines than the tetrahydronaphthalene derivative $\mathbf{2 5}$. Such high cytotoxicity of $\mathbf{2 4}$ is attributed to the presence nitrogen rich pyridazine moiety. From Table 1 it is noticed that all tested compounds showed very low cytotoxicity towards the normal cell line WI38 and such cytotoxicity levels are multiples of $10^{-4} \mathrm{nM}$.

\section{Toxicity}

Bioactive compounds are often toxic to shrimp larvae. Thus, in order to monitor these chemicals' in vivo lethality to shrimp larvae (Artemiasalina), Brine-Shrimp Lethality Assay [18] [19] was used. Results were analyzed with $\mathrm{LC}_{50}$ program to determine $\mathrm{LC}_{50}$ values and 95\% confidence intervals [20]. Results are given in Table 2 for the compounds which exhibited optimal cytotoxic effect against cancer cell lines which are the twelve compounds $3 \mathbf{b}, \mathbf{5 c}, \mathbf{7 b}, \mathbf{1 0 b}, \mathbf{1 2}, \mathbf{1 4 b}, \mathbf{1 6}, \mathbf{1 8 b}, \mathbf{1 9 b}, \mathbf{2 0 b}, 21$ and $\mathbf{2 4}$. The shrimp lethality assay is considered as a useful tool for preliminary assessment of toxicity, and it has been used for the detection of fungal toxins, plant extract toxicity, heavy metals, cyanobacteria toxins, pesticides, and cytotoxicity testing of dental materials [21], natural and syntheticorganic compounds. It has also been shown that, $A$. salina toxicity test results have a correlation with rodent and human acute oral toxicity data. Generally, a good correlation was obtained between $\mathrm{A}$. salina toxicity test and the rodent data. Likewise, the predictive screening potential of the aquatic invertebrate tests for acute oral toxicity in man, including A. salina toxicity test, was slightly better than the rat test for test compounds [22].

In order to prevent the toxicity results from possible false effects originated from solubility of compounds and DMSO's possible toxicity effect, compounds were prepared by dissolving in DMSO in the suggested DMSO volume ranges. It is clear from Table 2 that the 3-phenyl-2-thioxo-2,3,5,6-tetrahydrobenzo[d]thiazol-7(4H)-one (16) and the 2-amino-5-oxo-4-(2-phenylhydrazono)-4,5,6,7-tetrahydrobenzo[b]-thiophene-3-carbonitrile (21) showed non toxicity against the tested organisms.

\section{Experimental}

${ }^{13} \mathrm{C}$ NMR and ${ }^{1} \mathrm{H}$ NMR spectra were recorded on Bruker DPX400 instrument in DMSO with TMS as internal standard for protons and solvent signals as internal standard for carbon spectra. Chemical shift values are mentioned in $\delta$ (ppm). Mass spectra were recorded on EIMS (Shimadzu) and ESI-esquire 3000 Bruker Daltonics instrument. Elemental analyses were carried out by the Microanalytical Data Unit Ludwig-Maximilians-Universitat-Munchen, Germany. The progress of all reactions was monitored by TLC on $2 \times 5 \mathrm{~cm}$ pre-coated silica gel 60 F254 plates of thickness of $0.25 \mathrm{~mm}$ (Merck).

2-Benzylidenecyclohexane-1,3-dione (3a) and 2-(4-methoxybenzy-lidene)-cyclohexane-1,3-dione (3b)

General procedure: To a solution of cyclohexan-1,3-dione (1.12 g, $0.01 \mathrm{~mol})$ in 1,4-dioxane ( $40 \mathrm{~mL})$ containing piperidine $(0.50 \mathrm{~mL})$ either benzaldehyde $(1.06 \mathrm{~g}, 0.01 \mathrm{~mol})$ or p-methoxybenzaldehyde $(1.36 \mathrm{~g}, 0.01 \mathrm{~mol})$ was added. The reaction mixture, in each case, was heated under reflux for $2 \mathrm{~h}$ then poured onto ice water containing few drops of hydrochloric acid and the formed solid product was collected by filtration.

Compound 3a: White crystals (EtOH), yield $86 \%(1.60 \mathrm{~g}), \mathrm{mp} 205^{\circ} \mathrm{C}-207^{\circ} \mathrm{C}$. IR $(\mathrm{KBr}) \mathrm{cm}^{-1}: 3067,1722$, 1664, 1605. ${ }^{1} \mathrm{H}$ NMR (DMSO-d $\left.\mathrm{d}_{6}, 400 \mathrm{MHz}\right): \delta=1.82-1.88\left(\mathrm{~m}, 4 \mathrm{H}, 2 \mathrm{CH}_{2}\right), 2.24-2.26\left(\mathrm{~m}, 2 \mathrm{H}, \mathrm{CH}_{2}\right), 6.68(\mathrm{~s}$, $1 \mathrm{H}, \mathrm{CH}), 7.29-7.38$ (m, 5H, $\left.\mathrm{C}_{6} \mathrm{H}_{5}\right) .{ }^{13} \mathrm{C}$ NMR (DMSO-d 6 , $\left.75 \mathrm{MHz}\right): \delta=22.1,25.7,39.6\left(3^{-} \mathrm{CH}_{2}\right), 110.0(\mathrm{CH})$, 124.1, 124.9, 128.7, $139.5\left(\mathrm{C}_{6} \mathrm{H}_{5}\right), 139.9(\mathrm{C}=\mathrm{CH}), 181.1,189.5(2 \mathrm{C}=\mathrm{O})$. EIMS m/z $200[\mathrm{M}]^{+}(15)$; Analysis Calcd for $\mathrm{C}_{13} \mathrm{H}_{12} \mathrm{O}_{2}$ (200.23): C, 77.98; H, 6.04. Found: C, 78.21; H, 6.33.

Compound 3b: Pale yellow crystals $(\mathrm{EtOH})$, yield $82 \%(1.90 \mathrm{~g}), \mathrm{mp} 218^{\circ} \mathrm{C}-220^{\circ} \mathrm{C}$. IR $(\mathrm{KBr}) \mathrm{cm}^{-1}: 3023$, 2954, 2889, 1722, 1644, 1605. ${ }^{1} \mathrm{H}$ NMR (DMSO-d $\left.\mathrm{d}_{6}, 400 \mathrm{MHz}\right): \delta=1.62-1.93$ (m, 4H, 2CH${ }_{2}$ ), $2.24-2.26$ (m, $\left.2 \mathrm{H}, \mathrm{CH}_{2}\right), 3.68\left(\mathrm{~s}, 3 \mathrm{H}, \mathrm{CH}_{3}\right), 6.68(\mathrm{~s}, 1 \mathrm{H}, \mathrm{CH}), 7.24-7.36\left(2 \mathrm{~d}, 4 \mathrm{H}, \mathrm{J}=4.68 \mathrm{~Hz}, \mathrm{C}_{6} \mathrm{H}_{4}\right) .{ }^{13} \mathrm{C} \mathrm{NMR}\left(\mathrm{DMSO}-\mathrm{d}_{6}, 75\right.$ MHz): $\delta=22.2$, 25.9, $39.4\left(3 \mathrm{CH}_{2}\right), 36.6\left(\mathrm{CH}_{3}\right), 110.1(\mathrm{CH}), 124.0,124.9,128.8,139.6\left(\mathrm{C}_{6} \mathrm{H}_{5}\right), 139.6(\underline{\mathrm{C}}=\mathrm{CH})$, 181.0, $189.3(2 \mathrm{C}=\mathrm{O})$. EIMSm/z $230[\mathrm{M}]^{+}(20)$; Analysis Calcd for $\mathrm{C}_{14} \mathrm{H}_{14} \mathrm{O}_{3}(230.26)$ : C, 73.03; $\mathrm{H}, 6.13 \%$. Found: C, 73.31; H, 6.47\%. 
Table 2. Toxicity assay.

\begin{tabular}{|c|c|c|c|c|c|c|}
\hline Compound No. & Cons. $(\mu \mathrm{g} / \mathrm{ml})$ & Mortality $^{\mathrm{a}}$ & Toxicity & $\mathrm{LC}_{50}$ & Upper 95\% lim. & Lower 95\% lim. \\
\hline Зa & $\begin{array}{c}10 \\
100 \\
1000\end{array}$ & $\begin{array}{c}0 \\
3 \\
10\end{array}$ & Harmful & 316.23 & 255.25 & 111.43 \\
\hline $5 c$ & $\begin{array}{c}10 \\
100 \\
1000\end{array}$ & $\begin{array}{l}0 \\
4 \\
8\end{array}$ & Harmful & 17.78 & 860.12 & 180.30 \\
\hline $7 \mathbf{b}$ & $\begin{array}{c}10 \\
100 \\
1000\end{array}$ & $\begin{array}{c}0 \\
5 \\
10\end{array}$ & Harmful & 251.19 & 70.22 & 4.25 \\
\hline $10 \mathrm{~b}$ & $\begin{array}{c}10 \\
100 \\
1000\end{array}$ & $\begin{array}{c}0 \\
2 \\
10\end{array}$ & Harmful & 115.85 & 250.37 & 88.20 \\
\hline 12 & $\begin{array}{c}10 \\
100 \\
1000\end{array}$ & $\begin{array}{l}0 \\
0 \\
5\end{array}$ & Harmful & 100.00 & 104.2 & 157.62 \\
\hline $14 \mathrm{~b}$ & $\begin{array}{c}10 \\
100 \\
1000\end{array}$ & $\begin{array}{c}0 \\
1 \\
10\end{array}$ & Harmful & 87.78 & 320.40 & - \\
\hline 16 & $\begin{array}{c}10 \\
100 \\
1000\end{array}$ & $\begin{array}{l}0 \\
0 \\
3\end{array}$ & Non-toxic & 960.27 & - & - \\
\hline $18 b$ & $\begin{array}{c}10 \\
100 \\
1000\end{array}$ & $\begin{array}{c}5 \\
10 \\
10\end{array}$ & Very toxic & 10.00 & - & - \\
\hline $19 b$ & $\begin{array}{c}10 \\
100 \\
1000\end{array}$ & $\begin{array}{c}4 \\
10 \\
10\end{array}$ & Very toxic & 15.19 & - & - \\
\hline $20 \mathrm{~b}$ & $\begin{array}{c}10 \\
100 \\
1000\end{array}$ & $\begin{array}{l}0 \\
2 \\
8\end{array}$ & Harmful & 622.45 & 130.20 & 157.40 \\
\hline 21 & $\begin{array}{c}10 \\
100 \\
1000\end{array}$ & $\begin{array}{l}0 \\
0 \\
5\end{array}$ & Non-toxic & 1000.0 & - & - \\
\hline 24 & $\begin{array}{c}10 \\
100 \\
1000\end{array}$ & $\begin{array}{l}0 \\
5 \\
8\end{array}$ & Harmful & 614.68 & 650.37 & 166.20 \\
\hline
\end{tabular}

${ }^{\mathrm{a}}$ Ten organisms (A. salina) tested for each concentration.

2-Amino-4-benzylidene-5-oxo-4,5,6,7-tetrahydrobenzo[b]thiophene-3-carbonitrile (5a), ethyl 2-amino-4-benzylidene-5-oxo-4,5,6,7-tetrahydrobenzo[b]thiophene-3-carboxylate (5b), 2-amino-4-(p-methoxybenzylidene)-5-oxo4,5,6,7-tetrahydrobenzo[b]thiophene-3-carbonitrile (5c) and ethyl 2-amino-4-(p-methoxybenzylidene)-5-oxo-4,5,6,7tetrahydrobenzo[b]thiophene-3-carboxylate (5d)

General procedure: To a solution of either $3 \mathbf{a}(2.00 \mathrm{~g}, 0.01 \mathrm{~mol})$ or $\mathbf{3 b}(2.30 \mathrm{~g}, 0.01 \mathrm{~mol})$ in absolute ethanol $(50 \mathrm{~mL})$ containing triethylamine $(0.50 \mathrm{~mL})$ and elemental sulfur $(0.32 \mathrm{~g}, 0.01 \mathrm{~mol})$ either malononitrile $(0.66 \mathrm{~g}$, $0.01 \mathrm{~mol})$ or ethyl cyanoacetate $(1.13 \mathrm{~g}, 0.01 \mathrm{~mol})$ was added. The reaction mixture, in each case was heated under reflux for $2 \mathrm{~h}$ then was left to cool and the formed solid product, in each case, was collected by filtration.

Compound 5a: Yellow crystals $(\mathrm{EtOH})$, yield $77 \%(2.16 \mathrm{~g}), \mathrm{mp} 138^{\circ} \mathrm{C}-140^{\circ} \mathrm{C}$. IR $(\mathrm{KBr}) \mathrm{cm}^{-1}: 3466,3365$, 3055, 2948, 2222, 1720, 1657. ${ }^{1} \mathrm{H}$ NMR (DMSO, $\left.400 \mathrm{MHz}\right): \delta=1.84-1.93$ (m, 2H, $\mathrm{CH}_{2}$ ), $2.26-2.28$ (m, 2H, $\mathrm{CH}_{2}$ ), 4.55 (s, 2H, $\mathrm{D}_{2} \mathrm{O}$ exchangeable, $\mathrm{NH}_{2}$ ), $7.16(\mathrm{~s}, 1 \mathrm{H}, \mathrm{CH}), 7.19-7.27\left(\mathrm{~m}, 5 \mathrm{H}, \mathrm{C}_{6} \mathrm{H}_{5}\right) .{ }^{13} \mathrm{C}$ NMR (DMSO, 75 
MHz) $\delta$ : 23.6, $28.8\left(\mathrm{CH}_{2}\right), 110.2(\underline{\mathrm{CH}}=\mathrm{C}), 121.8(\mathrm{CN}), 124.1,125.8,128.4,129.2,130.3,137.3,140.2\left(\mathrm{C}_{6} \mathrm{H}_{5}\right.$, thiophene C), $147.5(\underline{C}=C H), 163.8(\mathrm{C}=\mathrm{O})$. EIMS m/z280 [M] $]^{+}(22)$; Analysis Calcd for $\mathrm{C}_{16} \mathrm{H}_{12} \mathrm{~N}_{2} \mathrm{OS}(280.34)$ : C, 68.55; H, 4.31; N, 9.99; S, 11.44. Found: C, 68.77; H, 4.29; N, 10.32; S, 11.61.

Compound 5b: Yellow crystals (EtOH), yield $84 \%(2.74 \mathrm{~g}), \mathrm{mp} 228^{\circ} \mathrm{C}-230^{\circ} \mathrm{C}$. IR $(\mathrm{KBr}) \mathrm{cm}^{-1}: 3478,3332$, 3050, 2950, 1720, 1688, 1653. ${ }^{1} \mathrm{H}$ NMR (DMSO-d 6 , $400 \mathrm{MHz}$ ): $\delta=1.27$ (t, 3H, J = 7.02 Hz, CH CH$_{3}, 1.92-1.94$ (m, $2 \mathrm{H}, \mathrm{CH}_{2}$ ), $2.25-2.28\left(\mathrm{~m}, 2 \mathrm{H}, \mathrm{CH}_{2}\right.$ ), 4.25 (q, $2 \mathrm{H}, \mathrm{J}=7.02 \mathrm{~Hz}, \mathrm{CH}_{2}$ ), 4.52 (s, $2 \mathrm{H}, \mathrm{D}_{2} \mathrm{O}$ exchangeable, $\mathrm{NH}_{2}$ ), $6.74(\mathrm{~s}, 1 \mathrm{H}, \mathrm{CH}), 7.14-8.07\left(\mathrm{~m}, 5 \mathrm{H}, \mathrm{C}_{6} \mathrm{H}_{5}\right) .{ }^{13} \mathrm{C}$ NMR (DMSO-d 6 , $\left.75 \mathrm{MHz}\right): \delta=13.6$ (ester $\left.\mathrm{CH}_{3}\right), 24.7,28.8$ $\left(\mathrm{CH}_{2}\right)$, 55.4 (ester $\left.\mathrm{CH}_{2}\right), 110.0(\underline{\mathrm{CH}}=\mathrm{C}), 124.3,125.8,128.6,129.0,130.8,137.3,140.0\left(\mathrm{C}_{6} \mathrm{H}_{5}\right.$, thiophene $\left.\mathrm{C}\right)$, $147.8(\mathrm{C}=\mathrm{CH}), 164.3,180.2(2 \mathrm{C}=\mathrm{O})$. EIMSm/z $327[\mathrm{M}]^{+}(18)$; Analysis Calcd for $\mathrm{C}_{18} \mathrm{H}_{17} \mathrm{NO}_{3} \mathrm{~S}$ (327.40): C, 66.03; H, 5.23; N, 4.28; S, 9.79. Found: C, 65.83; H, 5.08; N, 4.47; S, 7.63.

Compound 5c: Orange crystals from $(\mathrm{EtOH})$, yield $73 \%(2.26 \mathrm{~g}), \mathrm{mp} 222^{\circ} \mathrm{C}-225^{\circ} \mathrm{C}$. IR $(\mathrm{KBr}) \mathrm{cm}^{-1}$ : 3466 , 3368, 3058, 2938, 2893, 2220, 1720, 1644. ${ }^{1} \mathrm{H}$ NMR (DMSO-d $\left.\mathrm{d}_{6}, 400 \mathrm{MHz}\right): \delta=1.83-1.24$ (m, 2H, $\mathrm{CH}_{2}$ ), $2.58-$ 2.60 (m, 2H, $\mathrm{CH}_{2}$ ), 3.24 (s, 3H, $\mathrm{CH}_{3}$ ), 4.52 (s, 2H, $\mathrm{D}_{2} \mathrm{O}$ exchangeable, $\mathrm{NH}_{2}$ ), 6.74 (s, 1H, $\mathrm{CH}$ ), 7.03 - 7.35 (2dd, $\left.4 \mathrm{H}, \mathrm{J}=5.26 \mathrm{~Hz}, \mathrm{C}_{6} \mathrm{H}_{4}\right) .{ }^{13} \mathrm{C}$ NMR $\left(\mathrm{DMSO}_{6}, 75 \mathrm{MHz}\right): \delta=23.8$, $28.6\left(3 \mathrm{CH}_{2}\right), 36.9\left(\mathrm{CH}_{3}\right), 110.0(\underline{\mathrm{CH}}=\mathrm{C})$, $120.2(\mathrm{CN}), 124.3,125.9,128.4,129.0,130.3,137.5,140.6\left(\mathrm{C}_{6} \mathrm{H}_{5}\right.$, thiophene $\left.\mathrm{C}\right), 147.2(\mathrm{C}=\mathrm{CH}), 164.2(\mathrm{C}=\mathrm{O})$. EIMS m/z 310 [M] $]^{+}$(38); Analysis Calcd for $\mathrm{C}_{17} \mathrm{H}_{14} \mathrm{~N}_{2} \mathrm{O}_{2} \mathrm{~S}$ (310.37): C, 65.79; H, 4.55; N, 9.03; S, 10.33. Found: C, 65.88; H, 4.72; N, 8.79; S, 10.06.

Compound 5d: Yellow crystals (EtOH), yield $76 \%(2.71 \mathrm{~g}), \mathrm{mp} 140-142^{\circ} \mathrm{C}$. IR $(\mathrm{KBr}) \mathrm{cm}^{-1}: 3478,3328$, 3053, 2949, 2838, 1718, 1687, 1593. ${ }^{1} \mathrm{H}$ NMR (DMSO-d ${ }_{6}, 400 \mathrm{MHz}$ ): $\delta=1.22$ (t, 3H, J $=6.99 \mathrm{~Hz}, \mathrm{CH}_{3}$ ), $1.66-$ $1.74\left(\mathrm{~m}, 2 \mathrm{H}, \mathrm{CH}_{2}\right), 2.23$ - $2.32\left(\mathrm{~m}, 2 \mathrm{H}, \mathrm{CH}_{2}\right), 4.24\left(\mathrm{q}, 2 \mathrm{H}, \mathrm{J}=6.99 \mathrm{~Hz}, \mathrm{CH}_{2}\right), 4.50\left(\mathrm{~s}, 2 \mathrm{H}, \mathrm{D}_{2} \mathrm{O}\right.$ exchangeable, $\mathrm{NH}_{2}$ ), 6.79 (s, $\left.1 \mathrm{H}, \mathrm{CH}\right), 7.21-7.24$ (2dd, $4 \mathrm{H}, \mathrm{J}=4.86 \mathrm{~Hz}, \mathrm{C}_{6} \mathrm{H}_{4}$ ). ${ }^{13} \mathrm{C}$ NMR (DMSO-d 6 , 75Mz): $\delta=13.8$ (ester $\left.\mathrm{CH}_{3}\right)$, 24.6, 28.8, $60.0\left(3 \mathrm{CH}_{2}\right), 55.8\left(\right.$ ester $\left.\mathrm{CH}_{2}\right), 110.2(\mathrm{CH}=\mathrm{C}), 124.6,125.8,128.8,129.6,130.6,137.6,140.2$ $\left(\mathrm{C}_{6} \mathrm{H}_{5}\right.$, thiophene C), $147.6(\underline{\mathrm{C}}=\mathrm{CH}), 164.6(\mathrm{C}=\mathrm{O})$. EIMSm/z $357[\mathrm{M}]^{+}(12)$; Analysis Calcd for $\mathrm{C}_{19} \mathrm{H}_{19} \mathrm{NO}_{4} \mathrm{~S}$ (357.42): C, 63.85; H, 5.36; N, 3.92; S, 8.97. Found: C, 63.01; H, 5.22; N, 4.21; S, 9.27.

2-(Amino(2-amino-4-benzylidene-5-oxo-4,5,6,7-tetrahydrobenzo-[b]thiophen-3-yl)methylene)malononitrile (7a) and 2-(Amino(2-amino-4-(4-methoxybenzylidene)-5-oxo-4,5,6,7-tetrahydrobenzo[b]thiophen-3-yl) methylene) malononitrile $(\mathbf{7 b})$

General procedure: To a solution of either $3 \mathbf{a}(2.00 \mathrm{~g}, 0.01 \mathrm{~mol})$ or $3 \mathbf{b}(2.30 \mathrm{~g}, 0.01 \mathrm{~mol})$ in 1,4-dioxane (50 $\mathrm{mL}$ ) containing triethylamine $(0.50 \mathrm{~mL})$ and elemental sulfur $(0.32 \mathrm{~g}, 0.01 \mathrm{~mol})$, compound 6 (1.33 g, $0.01 \mathrm{~mol})$ was added. The reaction mixture was heated under reflux for $2 \mathrm{~h}$ then poured onto ice/water mixture containing few drops of hydrochloric acid (till $\mathrm{pH} 6$ ) was collected by filtration.

Compound 7a: Yellow crystals (AcOH), yield $80 \%(2.77 \mathrm{~g}), \mathrm{mp} 180^{\circ} \mathrm{C}-183^{\circ} \mathrm{C}$. IR $(\mathrm{KBr}) \mathrm{cm}^{-1}$ : 3488 - 3328, 3053, 2960, 2222, 2203, 1721, 1635. ${ }^{1} \mathrm{H}$ NMR (DMSO-d 6 , $400 \mathrm{MHz}$ ): $\delta=1.84-1.87$ (m, 2H, $\mathrm{CH}_{2}$ ), $2.42-2.48$ (m, $\left.2 \mathrm{H}, \mathrm{CH}_{2}\right)$, 3.90, $4.58\left(2 \mathrm{~s}, 4 \mathrm{H}, \mathrm{D}_{2} \mathrm{O}\right.$ exchangeable, $\left.2 \mathrm{NH}_{2}\right), 7.07(\mathrm{~s}, 1 \mathrm{H}, \mathrm{CH}), 7.13-7.29\left(\mathrm{~m}, 5 \mathrm{H}, \mathrm{C}_{6} \mathrm{H}_{5}\right) .{ }^{13} \mathrm{C}$ NMR (DMSO-d 6 , $75 \mathrm{MHz}): \delta=24.6,28.8\left(2 \mathrm{CH}_{2}\right), 110.2(\underline{\mathrm{CH}}=\mathrm{C}), 117.5,118.3(2 \mathrm{CN}), 119.2,120.4,124.8$, 140.3, 144.8, $145.0\left(\mathrm{C}_{6} \mathrm{H}_{5}\right.$, thiophene $\left.\mathrm{C}\right), 153.5(\underline{\mathrm{C}}=\mathrm{CH}), 167.3(\mathrm{C}=\mathrm{O}) . \mathrm{EIMSm} / \mathrm{z} 346[\mathrm{M}]^{+}(28)$; Analysis Calcd for $\mathrm{C}_{19} \mathrm{H}_{14} \mathrm{~N}_{4} \mathrm{OS}$ (346.41): C, 65.88; H, 4.07; N, 16.17; S, 9.26. Found: C, 65.79; H, 3.89; N, 16.09; S, 9.22.

Compound 7b: Orange crystals (AcOH), yield $84 \%(3.16 \mathrm{~g}), \mathrm{mp} 201^{\circ} \mathrm{C}-203^{\circ} \mathrm{C} . \mathrm{IR} \mathrm{cm}^{-1}: 3745-3390,3066$, 2951, 2840, 2220, 2212, 1722, 1660. ${ }^{1} \mathrm{H}$ NMR (DMSO-d $\left.\mathrm{d}_{6}, 400 \mathrm{MHz}\right): \delta=1.80-1.88$ (m, 2H, CH${ }_{2}$ ), $2.44-2.52$ (m, $\left.2 \mathrm{H}, \mathrm{CH}_{2}\right), 3.21\left(\mathrm{~s}, 3 \mathrm{H}, \mathrm{CH}_{3}\right), 3.84,4.52\left(2 \mathrm{~s}, 4 \mathrm{H}, \mathrm{D}_{2} \mathrm{O}\right.$ exchangeable, $\left.2 \mathrm{NH}_{2}\right), 6.88(\mathrm{~s}, 1 \mathrm{H}, \mathrm{CH}), 7.18-7.35$ (2dd, $\left.4 \mathrm{H}, \mathrm{J}=6.01 \mathrm{~Hz}, \mathrm{C}_{6} \mathrm{H}_{4}\right) .{ }^{13} \mathrm{C}$ NMR (DMSO-d 6 , $\left.75 \mathrm{MHz}\right): \delta=24.9,28.6\left(\mathrm{CH}_{2}\right), 55.8\left(\mathrm{CH}_{3}\right), 110.2(\underline{\mathrm{CH}}=\mathrm{C})$, 117.8, $118.8(2 \mathrm{CN}), 119.0,120.8,124.6,140.8,144.9,145.2\left(\mathrm{C}_{6} \mathrm{H}_{5}\right.$, thiophene $\left.\mathrm{C}\right), 153.8(\underline{\mathrm{C}}=\mathrm{CH}), 168.0(\mathrm{C}=\mathrm{O})$. EIMSm/z 376 [M] $]^{+}$(20); Analysis Calcd for $\mathrm{C}_{20} \mathrm{H}_{16} \mathrm{~N}_{4} \mathrm{O}_{2} \mathrm{~S}$ (376.43): C, 63.81; H, 4.28; N, 14.88; S, 8.52. Found: C, 63.56; H, 4.48; N, 15.04 .

2,4-diamino-8-oxo-5,6,7,8-tetrahydrobenzo[4,5]thieno[2,3-b]pyridine-3-carboxylate (9)

To a solution of compound $1(1.12 \mathrm{~g}, 0.01 \mathrm{~mol})$ in 1,4-dioxane $(50 \mathrm{~mL})$ containing triethylamine $(0.50 \mathrm{~mL})$ and elemental sulfur $(0.32 \mathrm{~g}, 0.01 \mathrm{~mol})$ compound 6 ( $1.33 \mathrm{~g}, 0.01 \mathrm{~mol})$ was added. The reaction mixture was heated under reflux for $2 \mathrm{~h}$ then poured onto ice/water mixture containing few drops of hydrochloric acid (till $\mathrm{pH}$ 6) was collected by filtration.

Compound 9: Yellow crystals (AcOH), yield 65\% (1.67 g), mp $166^{\circ} \mathrm{C}-168^{\circ} \mathrm{C}$. IR $(\mathrm{KBr}) \mathrm{cm}^{-1}$ : 3588 - 3329, 3034, 2925, 2185, 1720, 1633. ${ }^{1} \mathrm{H}$ NMR (DMSO-d $\left.\mathrm{d}_{6}, 400 \mathrm{MHz}\right): \delta=1.81-1.88$ (m, 4H, 2CH $), 2.49-2.51(\mathrm{~m}$, $2 \mathrm{H}, \mathrm{CH}_{2}$ ), 3.56, $4.71\left(2 \mathrm{~s}, 4 \mathrm{H}, \mathrm{D}_{2} \mathrm{O}\right.$ exchangeable, $\left.2 \mathrm{NH}_{2}\right) .{ }^{13} \mathrm{C}$ NMR (DMSO-d $\left.\mathrm{d}_{6}, 75 \mathrm{MHz}\right): \delta=20.1$, 32.2, 32.3 (3 $\left.\mathrm{CH}_{2}\right), 116.0(\mathrm{CN}), 129.6,133.8,138.9,140.3,144.6,146.0$ (thiophene, pyridine $\left.\mathrm{C}\right), 168.2(\mathrm{C}=\mathrm{O}), 174.4(\mathrm{C}=\mathrm{N})$. 
EIMS m/z $258[\mathrm{M}]^{+}$(20); Analysis Calcd for $\mathrm{C}_{12} \mathrm{H}_{10} \mathrm{~N}_{4} \mathrm{OS}$ (258.30): C, 55.80; H, 3.90; N, 21.69; S, 12.41 . Found: C, 55.69; H, 4.18; N, 21.82; S, 12.29 .

2-Amino-5,6,7,8-tetrahydro-5-oxo-4-phenyl-4H-chromene-3-carbonitrile (10a) and 2-hydroxy-5,6,7,8-tetrahydro-5-oxo-4-phenyl-4H-chromene-3-carbonitrile (10b)

General procedure: Method (A): To a solution of either compound 3a (2.00 g, $0.01 \mathrm{~mol})$ or $\mathbf{3 b}(2.30 \mathrm{~g}, 0.01$ $\mathrm{mol})$ in 1,4-dioxane $(50 \mathrm{~mL})$ containingtriethylamine $(0.50 \mathrm{~mL})$ either malononitrile $(0.66 \mathrm{~g}, 0.01 \mathrm{~mol})$ or ethyl cyanoacetate $(1.13 \mathrm{~g}, 0.01 \mathrm{~mol})$ was added. The reaction mixture was heated under reflux for $4 \mathrm{~h}$ then poured onto ice/water and the formed solid product was collected by filtration.

Method B: To a solution of compound $1(1.12 \mathrm{~g}, 0.01 \mathrm{~mol})$ in ethanol $(50 \mathrm{~mL})$ containing triethylamine $(0.50$ $\mathrm{mL}$ ) either $\alpha$-cyanocinnamonitrile (1.54 g, $0.01 \mathrm{~mol})$, ethyl $\alpha$-cyanocinnamate ( $2.02 \mathrm{~g}, 0.01 \mathrm{~mol})$ was added. The reaction mixture, in each case, was heated under reflux for $4 \mathrm{~h}$ then poured onto ice/water and the formed solid product was collected by filtration.

Compound 10a: Pall yellow crystals (EtOH), yield 73\% (1.94 g), mp $233^{\circ} \mathrm{C}-235^{\circ} \mathrm{C}$. IR $(\mathrm{KBr}) \mathrm{cm}^{-1}$ : 3583 3322, 3052, 2925, 2190, 1680, 1635. ${ }^{1} \mathrm{H}$ NMR (DMSO-d $\left.\mathrm{d}_{6}, 400 \mathrm{MHz}\right): \delta=1.90-1.96\left(\mathrm{~m}, 4 \mathrm{H}, 2 \mathrm{CH}_{2}\right), 2.24-$ $2.51\left(\mathrm{~m}, 2 \mathrm{H}, \mathrm{CH}_{2}\right), 4.19\left(\mathrm{~s}, 2 \mathrm{H}, \mathrm{D}_{2} \mathrm{O}\right.$ exchangeable, $\left.\mathrm{NH}_{2}\right), 7.01(\mathrm{~s}, 1 \mathrm{H}$, pyran $\mathrm{H}-4), 7.17-7.31\left(\mathrm{~m}, 5 \mathrm{H}, \mathrm{C}_{6} \mathrm{H}_{5}\right)$. ${ }^{13} \mathrm{C}$ NMR (DMSO-d 6 , 75 MHz): $\delta=20.3$, 27.0, $36.0\left(3 \mathrm{CH}_{2}\right), 114.3$ (pyran C-4), $120.3(\mathrm{CN}), 120.3,127.7,128.8$, 128.9, 129.0, 140.7, $159.0\left(\mathrm{C}_{6} \mathrm{H}_{5}\right.$, pyran C), $196.4(\mathrm{C}=\mathrm{O})$. EIMS m/z $266[\mathrm{M}]^{+}(26)$; Analysis Calcd for $\mathrm{C}_{16} \mathrm{H}_{14} \mathrm{~N}_{2} \mathrm{O}_{2}$ (266.29): C, 72.16; H, 5.30; N, 10.52. Found: C, 72.38; H, 5.26; N, 10.47 .

Compound 10b: Yellow crystals $(\mathrm{EtOH})$, yield $60 \%(1.60 \mathrm{~g}), \mathrm{mp} 122-114^{\circ} \mathrm{C} . \mathrm{IR}(\mathrm{KBr}) \mathrm{cm}^{-1}: 3583-3322$, 3052, 2925, 2220, 1689, 1638. ${ }^{1} \mathrm{H}$ NMR (DMSO-d 6 , $\left.400 \mathrm{MHz}\right): \delta=1.91-1.96$ (m, 4H, 2CH $), 2.23-2.50(\mathrm{~m}$, $\left.2 \mathrm{H}, \mathrm{CH}_{2}\right), 6.99$ (s, $1 \mathrm{H}$, pyran H-4), $7.26-7.36\left(\mathrm{~m}, 5 \mathrm{H}, \mathrm{C}_{6} \mathrm{H}_{5}\right), 10.22(\mathrm{~s}, 1 \mathrm{H}, \mathrm{OH}) .{ }^{13} \mathrm{C}$ NMR (DMSO-d 6 , 75 MHz): $\delta=20.1,27.2$, $36.8\left(3 \mathrm{CH}_{2}\right), 114.0$ (pyran C-4), $120.1(\mathrm{CN}), 120.2,126.9,128.4,128.5,129.2,140.3$, $159.5\left(\mathrm{C}_{6} \mathrm{H}_{5}\right.$, pyran C), $194.2(\mathrm{C}=\mathrm{O})$. EIMSm/z $267[\mathrm{M}]^{+}$(18); Analysis Calcd for $\mathrm{C}_{16} \mathrm{H}_{13} \mathrm{NO}_{3}$ (267.28): C, 71.90; H, 4.90; N, 5.24. Found: C, 72.16; H, 5.21; N, 5.29.

2,4-Diamino-5-oxo-5,6,7,8-tetrahydronaphthalene-1,3-dicarbonitrile (12)

To a solution of compound $1(1.12 \mathrm{~g}, 0.01 \mathrm{~mol})$ in absolute ethanol $(40 \mathrm{~mL})$ containing triethylamine, compound 6 (1.33 g, $0.01 \mathrm{~mol}$ ) was added. The reaction mixture was heated under reflux for $4 \mathrm{~h}$ and the formed solid product upon cooling was collected by filtration.

Compound 12: Yellow crystals (EtOH), yield $80 \%(1.80 \mathrm{~g}), \mathrm{mp} 220^{\circ} \mathrm{C}-228^{\circ} \mathrm{C}$. IR $(\mathrm{KBr}) \mathrm{cm}^{-1}$ : 3339 - 3197, 2920, 2222, 2190, 1683, 1630. ${ }^{1} \mathrm{H}$ NMR (DMSO-d $\left.\mathrm{d}_{6}, 400 \mathrm{MHz}\right): \delta=1.68-1.71$ (m, 4H, 2CH ${ }_{2}$ ), $2.34-2.46$ (m, $\left.2 \mathrm{H}, \mathrm{CH}_{2}\right), 5.21,5.79\left(2 \mathrm{~s}, 4 \mathrm{H}, \mathrm{D}_{2} \mathrm{O}\right.$ exchangeable, $\left.2 \mathrm{NH}_{2}\right) .{ }^{13} \mathrm{C}$ NMR (DMSO-d 6 , $\left.75 \mathrm{MHz}\right): \delta=20.3,28.5,50.20$ $\left(3 \mathrm{CH}_{2}\right), 118.2$, $118.6(2 \mathrm{CN}), 119.8,120.6,124.4,129.6$ (benzene C), $196.8(\mathrm{C}=\mathrm{O})$. EIMSm/z $226[\mathrm{M}]^{+}(30)$; Analysis Calcd for $\mathrm{C}_{12} \mathrm{H}_{10} \mathrm{~N}_{4} \mathrm{O}$ (226.23): C, 63.71; H, 4.46; N, 24.76. Found: C, 63.58; H, 4.62; N, 24.41.

2,4-Diamino-8-benzylidene-7-oxo-5,6,7,8-tetrahydronaphthalene-1,3-dicarbonitrile (14a) and 2,4-diamino-8(4-methoxybenzylidene)-7-oxo-5,6,7,8-tetrahydronaphthalene-1,3-dicarbonitrile (14a)

General procedure: To a solution of either compound $3 \mathbf{a}(2.00 \mathrm{~g}, 0.01 \mathrm{~mol})$ or $3 \mathbf{b}(2.30 \mathrm{~g}, 0.01 \mathrm{~mol})$ in ethanol $(50 \mathrm{~mL})$ containing triethylamine $(0.50 \mathrm{~mL})$, compound $6(1.33 \mathrm{~g}, 0.01 \mathrm{~mol})$ was added. The reaction mixture was heated under reflux for $6 \mathrm{~h}$. The solid product, formed in each case, upon pouring onto ice/water mixture containing few drops of hydrochloric acid.

Compound 14a: Yellow crystals (1,4-dioxane), yield 77\% (2.42 g), mp $199^{\circ} \mathrm{C}-201^{\circ} \mathrm{C}$. IR $(\mathrm{KBr}) \mathrm{cm}^{-1}: 3742$ 3343, 3055, 2948, 2220, 2208, 1683, 1632. ${ }^{1} \mathrm{H}$ NMR (DMSO-d 6 , $\left.400 \mathrm{MHz}\right): \delta=1.84-1.96\left(\mathrm{~m}, 2 \mathrm{H}, \mathrm{CH}_{2}\right), 2.23$ - $2.50\left(\mathrm{~m}, 2 \mathrm{H}, \mathrm{CH}_{2}\right), 4.33,5.01\left(2 \mathrm{~s}, 4 \mathrm{H}, \mathrm{D}_{2} \mathrm{O}\right.$ exchangeable, $\left.2 \mathrm{NH}_{2}\right), 6.87(\mathrm{~s}, 1 \mathrm{H}, \mathrm{CH}=\mathrm{C}), 7.27-7.37(\mathrm{~m}, 5 \mathrm{H}$, $\left.\mathrm{C}_{6} \mathrm{H}_{5}\right) .{ }^{13} \mathrm{C}$ NMR (DMSO-d 6 , $\left.75 \mathrm{MHz}\right): \delta=20.6,36.8\left(2 \mathrm{CH}_{2}\right), 95.4,95.3(\mathrm{CH}=\mathrm{C}), 110.2(\mathrm{CH}=\mathrm{C}), 116.8,117.3$ (2CN), 127.7, 128.8, 128.9, 129.0, 129.2, 129.8, 140.7 (two benzene C), 196.4 (C=O). EIMSm/z 314 [M] ${ }^{+}$(38); Analysis Calcd for $\mathrm{C}_{19} \mathrm{H}_{14} \mathrm{~N}_{4} \mathrm{O}$ (314.34): C, 72.60; H, 4.49; N, 17.82. Found: C, 72.88; H, 4.69; N, 17.59 .

Compound 14b: White crystals $(\mathrm{EtOH})$, yield $68 \%(2.34 \mathrm{~g}), \mathrm{mp} 180^{\circ} \mathrm{C}-183^{\circ} \mathrm{C}$. IR $(\mathrm{KBr}) \mathrm{cm}^{-1}$ : 3577 - 3320 , 3050, 2920, 2218, 2201, 1677, 1636. ${ }^{1} \mathrm{H}$ NMR (DMSO-d $\left.{ }_{6}, 400 \mathrm{MHz}\right): \delta=1.83-1.94$ (m, 2H, CH${ }_{2}$ ), $2.28-2.51$ (m, 2H, $\mathrm{CH}_{2}$ ), 3.65 (s, $\left.3 \mathrm{H}, \mathrm{CH}_{3}\right), 3.51,4.65\left(2 \mathrm{~s}, 4 \mathrm{H}, \mathrm{D}_{2} \mathrm{O}\right.$ exchangeable $\left.2 \mathrm{NH}_{2}\right), 6.45(\mathrm{~s}, 1 \mathrm{H}, \mathrm{CH}=\mathrm{C}), 7.28$ - 7.38 (2dd, $\left.4 \mathrm{H}, \mathrm{J}=5.69 \mathrm{~Hz}, \mathrm{C}_{6} \mathrm{H}_{4}\right) .{ }^{13} \mathrm{C}$ NMR (DMSO-d $\left.\mathrm{d}_{6}, 75 \mathrm{MHz}\right): \delta=20.1,37.8\left(2 \mathrm{CH}_{2}\right), 55.9\left(\mathrm{OCH}_{3}\right), 95.2$ $(\underline{\mathrm{CH}}=\mathrm{C}), 110.2(\mathrm{CH}=\mathrm{C}), 118.0,118.3(2 \mathrm{CN}), 127.5,128.4,128.9,129.0,129.2,129.9,136.8,155.0$ (two benzene C), 194.3(C=O). EIMS m/z $344[\mathrm{M}]^{+}$(22); Analysis Calcd for $\mathrm{C}_{20} \mathrm{H}_{16} \mathrm{~N}_{4} \mathrm{O}_{2}$ (344.37): C, 69.76; $\mathrm{H}, 4.68 ; \mathrm{N}$, 16.27. Found: C, 70.03; H, 4.88; N, 16.17 .

3-Phenyl-2-thioxo-2,3,5,6-tetrahydrobenzo[d]thiazol-7(4H)-one (16) 
To a solution of compound 1 (1.12 g, $0.01 \mathrm{~mol})$ in 1,4-dioxane $(40 \mathrm{~mL})$ containing triethylamine, elemental sulfur $(0.32 \mathrm{~g}, 0.01 \mathrm{~mol})$ and phenylisothiocyanate $(1.30 \mathrm{~g}, 0.01 \mathrm{~mol})$ were added. The reaction mixture was heated under reflux for $2 \mathrm{~h}$ then left to cool. The formed solid product was collected by filtration.

Compound 16: Yellow crystals from 1,4-dioxane, yield $90 \%(2.35 \mathrm{~g}), \mathrm{mp} 240^{\circ} \mathrm{C}-244^{\circ} \mathrm{C}$. IR $(\mathrm{KBr}) \mathrm{cm}^{-1}$ : 3035, 2944, 1662, 1582. ${ }^{1} \mathrm{H}$ NMR (DMSO-d $\left.6,400 \mathrm{MHz}\right): \delta=2.00-2.03\left(\mathrm{~m}, 4 \mathrm{H}, \mathrm{CH}_{2}\right), 2.45-2.47(\mathrm{~m}, 2 \mathrm{H}$, $\mathrm{CH}_{2}$ ), $7.30-7.56\left(\mathrm{~m}, 5 \mathrm{H}, \mathrm{C}_{6} \mathrm{H}_{5}\right) .{ }^{13} \mathrm{C}$ NMR (DMSO-d 6 , $\left.75 \mathrm{MHz}\right): \delta=20.6,24.6,36.9\left(3 \mathrm{CH}_{2}\right), 127.7,128.9$, 129.2, 130.5, 136.8, 139.9, 157.8 (benzene, thiophene C), 180.1 (C=S), 191.34 (C=O). EIMSm/z 264 [M] ${ }^{+}$(22); Analysis Calcd for $\mathrm{C}_{13} \mathrm{H}_{11} \mathrm{NOS}_{2}$ (261.36): C, 59.74; H, 4.24; N, 5.36; S, 24.54. Found: C, 59.93; H, 4.29; N, 5.41; S, 24.36.

2-(2-Phenylhydrazono)cyclohexane-1,3-dione(18a) and 2-(2-4-chlorophenylhydrazono)cyclohexane-1,3-dione (18b)

General procedure: To a cold solution $\left(0^{\circ} \mathrm{C}-5^{\circ} \mathrm{C}\right)$ of compound $1(1.12 \mathrm{~g}, 0.01 \mathrm{~mol})$ in ethanol $(50 \mathrm{~mL})$ containing sodium acetate $(3.50 \mathrm{~g}, 0.50 \mathrm{~mol})$ either benzenediazonium chloride $(0.01 \mathrm{~mol})$ or 4-chlorobenzene-diazonium chloride $(0.01 \mathrm{~mol}$ [prepared by adding a cold solution of sodium nitrite $(0.70 \mathrm{~g}$, in water $(10 \mathrm{~mL}))$ to a cold solution $\left(0^{\circ} \mathrm{C}-5^{\circ} \mathrm{C}\right)$ of either aniline oil $(0.93 \mathrm{~g}, 0.01 \mathrm{~mol})$ or 4-chloroaniline $(1.27 \mathrm{~g}, 0.01 \mathrm{~mol})$ in concentrated hydrochloric acid $(12 \mathrm{~mL})$ with continuous stirring] was added with continuous stirring. The whole reaction mixture was left at room temperature for $1 \mathrm{~h}$ then the formed solid product was collected by filtration.

Compound 18a: Orange crystals from 1,4-dioxane, yield $82 \%(1.72 \mathrm{~g}), \mathrm{mp} 138^{\circ} \mathrm{C}-140^{\circ} \mathrm{C}$. IR $(\mathrm{KBr}) \mathrm{cm}^{-1}$ : 3540 - 3426, 3050, 2947, 1740, 1668, 1619. ${ }^{1} \mathrm{H}$ NMR (DMSO-d 6 , $\left.400 \mathrm{MHz}\right): \delta=1.93-1.96$ (m, 2H, $\mathrm{CH}_{2}$ ), 2.45 - 2.47 (m, 4H, 2CH $), 7.42-7.61$ (m, 5H, $\left.\mathrm{C}_{6} \mathrm{H}_{5}\right), 14.87$ (s, $\left.1 \mathrm{H}, \mathrm{NH}\right) .{ }^{13} \mathrm{C}$ NMR (DMSO-d 6 , $\left.75 \mathrm{MHz}\right): \delta=18.9$, 26.2 (3 $\left.\mathrm{CH}_{2}\right), 117.5,126.9,130.3,131.6$ (benzene C), $163.1(\mathrm{C}=\mathrm{N}), 196.4$ (2 C=O). EIMS m/z 216 [M] ${ }^{+}$(20); Analysis Calcd for $\mathrm{C}_{12} \mathrm{H}_{12} \mathrm{~N}_{2} \mathrm{O}_{2}$ (216.24): C, 66.65; H, 5.59; N, 12.96. Found: C, 66.84; H, 5.60; N, 13.28 .

Compound 18b: Orange crystals (EtOH), yield $90 \%(2.25 \mathrm{~g}), \mathrm{mp} 212^{\circ} \mathrm{C}-215^{\circ} \mathrm{C}$. IR $(\mathrm{KBr}) \mathrm{cm}^{-1}: 3540-3447$, 3049, 2946, 1720, 1663, 1619. ${ }^{1} \mathrm{H}$ NMR (DMSO-d $\left.\mathrm{d}_{6}, 400 \mathrm{MHz}\right): \delta=1.93-1.98\left(\mathrm{~m}, 2 \mathrm{H}, \mathrm{CH}_{2}\right), 2.45-2.68(\mathrm{~m}$, $4 \mathrm{H}, 2 \mathrm{CH}_{2}$ ), $7.48-7.62$ (2dd, $\left.4 \mathrm{H}, \mathrm{j}=6.02 \mathrm{~Hz}, \mathrm{C}_{6} \mathrm{H}_{4}\right), 14.70$ (s, $\left.1 \mathrm{H}, \mathrm{NH}\right) .{ }^{13} \mathrm{C}$ NMR (DMSO-d 6 , $75 \mathrm{Mz}$ ): $\delta=18.3$, $26.1\left(3 \mathrm{CH}_{2}\right), 119.3,130.1,130.6141 .5$ (benzene C), $198.2(\mathrm{C}=\mathrm{N}), 193.7(\mathrm{C}=\mathrm{O})$. EIMS: m/z $250[\mathrm{M}]^{+}(17)$. Analysis Calcd for $\mathrm{C}_{12} \mathrm{H}_{11} \mathrm{ClN}_{2} \mathrm{O}_{2}$ (250.68): C, 57.49; H, 4.42; N, 11.17. Found: C, 57.27; H, 4.77; N, 10.93.

2-(3-Oxo-2-(2-phenylhydrazono)cyclohexylidene)malononitrile (19a) and ethyl 2-cyano-2-(3-oxo-2-(2-phenyylhydrazono)cyclohexylidene)acetate (19b)

General procedure: To the dry solid of compound 18a $(2.16 \mathrm{~g}, 0.01 \mathrm{~mol})$ either malononitrile $(0.66 \mathrm{~g}, 0.01$ $\mathrm{mol})$ or ethyl cyanoacetate $(1.13 \mathrm{~g}, 0.01 \mathrm{~mol})$ and ammonium acetate $(0.50 \mathrm{~g})$ were added. The reaction mixture, in each case was heated in an oil bath at $120^{\circ} \mathrm{C}$ for $15 \mathrm{~min}$. The solid product produced after boiling with ethanol was collected by filtration.

Compound 19a: Yellowcrystals (EtOH), yield 78\% (2.06 g), mp $210^{\circ} \mathrm{C}-213^{\circ} \mathrm{C}$. IR $(\mathrm{KBr}) \mathrm{cm}^{-1}: 3544-3373$, 3033, 2934, 2222, 2220, 1722, 1632. ${ }^{1} \mathrm{H}$ NMR (DMSO-d $\left.\mathrm{d}_{6}, 400 \mathrm{MHz}\right): \delta=1.93-1.98$ (m, 2H, CH${ }_{2}$ ), $2.45-2.67$ (m, 4H, $\mathrm{CH}_{2}$ ), $7.42-7.59\left(\mathrm{~m}, 5 \mathrm{H}, \mathrm{C}_{6} \mathrm{H}_{5}\right), 14.86(\mathrm{~s}, 1 \mathrm{H}, \mathrm{NH}) .{ }^{13} \mathrm{C}$ NMR (DMSO-d 6 , $75 \mathrm{MHz}$ ): $\delta=18.29,33.2$, $39.2\left(3 \mathrm{CH}_{2}\right), 103.6,104.4(\mathrm{C}=\mathrm{C}), 114.5,117.6(2 \mathrm{CN}), 119.3,130.0,130.6141 .6$ (benzene $\left.\mathrm{C}\right), 151.4(\mathrm{C}=\mathrm{N})$, $162.8(\mathrm{C}=\mathrm{O})$. EIMSm/z $264[\mathrm{M}]^{+}$(23); Analysis Calcd for $\mathrm{C}_{15} \mathrm{H}_{12} \mathrm{~N}_{4} \mathrm{O}$ (264.28): C, 68.17; H, 4.58; N, 21.20 . Found: C, 68.37; H, 4.72; N, 21.09.

Compound 19b: Yellow crystals (EtOH), yield $70 \%(2.17 \mathrm{~g}), \mathrm{mp} 150^{\circ} \mathrm{C}-152^{\circ} \mathrm{C}$. IR $(\mathrm{KBr}) \mathrm{cm}^{-1}: 3522$ - 3329, 3038, 2936, 2212, 1720, 1687, 1630. ${ }^{1} \mathrm{H}$ NMR (DMSO-d 6 , $400 \mathrm{MHz}$ ): $\delta=1.19$ (t, 3H, J = 7.33 Hz, CH $\mathrm{CH}_{3}$ ), $1.87-$ $1.93\left(\mathrm{~m}, 2 \mathrm{H}, \mathrm{CH}_{2}\right), 2.43-2.65\left(\mathrm{~m}, 4 \mathrm{H}, 2 \mathrm{CH}_{2}\right), 4,22\left(\mathrm{q}, 2 \mathrm{H}, \mathrm{J}=7.33 \mathrm{~Hz}, \mathrm{CH}_{2}\right), 7.38-7.54\left(\mathrm{~m}, 5 \mathrm{H}, \mathrm{C}_{6} \mathrm{H}_{5}\right), 14.82$ (s, 1H, NH). ${ }^{13} \mathrm{C}$ NMR (DMSO-d 6 , $\left.75 \mathrm{MHz}\right): \delta=13.6\left(\mathrm{CH}_{3}\right), 18.29,33.0,39.4\left(3 \mathrm{CH}_{2}\right), 59.5\left(\mathrm{CH}_{2}\right), 103.8,104.9$ $(\mathrm{C}=\mathrm{C}), 116.5(\mathrm{CN}), 119.0,120.5,130.6,141.6$ (benzene $\mathrm{C}), 150.2(\mathrm{C}=\mathrm{N}), 160.8(\mathrm{C}=\mathrm{N}), 163.1,164.4(2 \mathrm{C}=\mathrm{O})$. EIMSm/z $311[\mathrm{M}]^{+}$(20); Analysis Calcd for $\mathrm{C}_{17} \mathrm{H}_{17} \mathrm{~N}_{3} \mathrm{O}_{3}$ (311.34): C, 65.58; H, 5.50; N, 13.50. Found: C, 65.88; H, 5.71; N, 13.69 .

3-Imino-8-oxo-2-phenyl-2,3,5,6,7,8-hexahydrocinnoline-4-carbonitrile (20a) and 3,8-dioxo-2-phenyl-2,3,5,6, 7,8-hexahydrocinnoline-4-carbonitrile (20b)

General procedure: To a solution of compound $18 \mathrm{a}(2.16 \mathrm{~g}, 0.01 \mathrm{~mol})$ in 1,4-dioxane (40 mL) containing triethylamine $(0.50 \mathrm{~mL})$ either malononitrile $(0.66 \mathrm{~g}, 0.01 \mathrm{~mol})$ or ethyl cyanoacetate $(1.13 \mathrm{~g}, 0.01 \mathrm{~mol})$ was added. The reaction mixture, in each case was heated under reflux for $3 \mathrm{~h}$ then evaporated under vacuum. The solid product produced after triturating the remaining product with diethyl ether was collected by filtration.

Compound 20a: Orange crystals (EtOH), yield $80 \%(2.11 \mathrm{~g}), \mathrm{mp} 150^{\circ} \mathrm{C}-153^{\circ} \mathrm{C}$. IR $(\mathrm{KBr}) \mathrm{cm}^{-1}: 3741$ - 3376, 
3055, 2933, 2193, 1737,1655, 1631. ${ }^{1} \mathrm{H}$ NMR (DMSO-d $\left.\mathrm{d}_{6}, 400 \mathrm{MHz}\right): \delta=1.94-1.98$ (m, 2H, CH CH $_{2}, 2.48-2.50$ (m, 4H, $\mathrm{CH}_{2}$ ), $7.45-7.67$ (m, 5H, $\mathrm{C}_{6} \mathrm{H}_{5}$ ), 8.09 (s, $1 \mathrm{H}, \mathrm{D}_{2} \mathrm{O}$ exchangeable, $\left.\mathrm{NH}\right) .{ }^{13} \mathrm{C}$ NMR (DMSO-d 6 , $75 \mathrm{MHz}$ ): $\delta=18.2,18.3,39.2\left(3 \mathrm{CH}_{2}\right), 103.6,104.4(\mathrm{C}=\mathrm{C}), 118.6(\mathrm{CN}), 119.0,130.3,130.8,133.4,138.3,141.9,142.0$ (benzene, pyridazine C), $173.2(\mathrm{C}=\mathrm{N}), 196.5(\mathrm{CO})$. EIMS m/z $264[\mathrm{M}]^{+}(20 \%)$; Analysis Calcd for $\mathrm{C}_{15} \mathrm{H}_{12} \mathrm{~N}_{4} \mathrm{O}$ (264.28): C, 68.17; H, 4.58; N, 21.20. Found: C, 68.28; H, 4.77; N, 21.08.

Compound 20b: Yellow crystals (EtOH), yield $69 \%(1.82 \mathrm{~g}), \mathrm{mp} 139^{\circ} \mathrm{C}-141^{\circ} \mathrm{C}$. IR $(\mathrm{KBr}) \mathrm{cm}^{-1}: 3055,2945$, 2212, 1693, 1684, 1630. ${ }^{1} \mathrm{H}$ NMR (DMSO-d $\left.6,400 \mathrm{MHz}\right): \delta=1.94-1.98\left(\mathrm{~m}, 2 \mathrm{H}, \mathrm{CH}_{2}\right), 2.49-2.68(\mathrm{~m}, 4 \mathrm{H}$, $\mathrm{CH}_{2}$ ), 7.45 - $7.61\left(\mathrm{~m}, 5 \mathrm{H}, \mathrm{C}_{6} \mathrm{H}_{5}\right) .{ }^{13} \mathrm{C}$ NMR (DMSO-d 6 , $\left.75 \mathrm{MHz}\right): \delta=18.3$, 33.0, $39.1\left(3 \mathrm{CH}_{2}\right), 117.5(\mathrm{CN}), 118.6$, 119.0, 120.3, 126.9, 130.6, 131.9, 141.6, 142.1 (benzene, pyridazine C), $168.3(\mathrm{C}=\mathrm{N}), 198.1$ (C=O). EIMSm/z $265[\mathrm{M}]^{+}$(28\%); Analysis Calcd for $\mathrm{C}_{15} \mathrm{H}_{11} \mathrm{~N}_{3} \mathrm{O}_{2}$ (265.27): C, 67.92; H, 4.18; N, 15.84. Found: C, 67.77; H, 4.30; N, 15.64 .

2-Amino-5-oxo-4-(2-phenylhydrazono)-4,5,6,7-tetrahydrobenzo[b]-thiophene-3-carbonitrile (21) and 4-amino-2-phenyl-6,7-dihydro-2H-thieno[4,3,2-de]cinnoline-3,8-dione (22)

General procedure: To a solution of compound 18a (2.16 g, $0.01 \mathrm{~mol})$ in 1,4-dioxane $(40 \mathrm{~mL})$ containing triethylamine $(0.50 \mathrm{~mL})$, either malononitrile $(0.66 \mathrm{~g}, 0.01 \mathrm{~mol})$ or ethyl cyanoacetate $(1.13 \mathrm{~g}, 0.01 \mathrm{~mol})$. The reaction mixture was heated under reflux for $2 \mathrm{~h}$ then poured onto ice/water and the formed solid product was collected by filtration.

Compound 21: Orange crystals (EtOH), yield $70 \%(2.07 \mathrm{~g}), \mathrm{mp} 138^{\circ} \mathrm{C}-140^{\circ} \mathrm{C}$. IR $(\mathrm{KBr}) \mathrm{cm}^{-1}$ : 3417 - 3320, 3053, 2947, 2197, 1742, 1634. ${ }^{1} \mathrm{H}$ NMR (DMSO-d 6 , $\left.400 \mathrm{MHz}\right): \delta=1.93-1.96$ (m, 2H, CH$\left.)_{2}\right), 2.46-2.50(\mathrm{~m}$, $2 \mathrm{H}, \mathrm{CH}_{2}$ ), 6.42 (s, 2H, $\mathrm{D}_{2} \mathrm{O}$ exchangeable, $\mathrm{NH}_{2}$ ), 7.22 - 7.57 (m, 5H, $\left.\mathrm{C}_{6} \mathrm{H}_{5}\right), 14.84$ (s, $1 \mathrm{H}, \mathrm{D}_{2} \mathrm{O}$ exchangeable, $\mathrm{NH}){ }^{13}{ }^{\mathrm{C}}$ NMR (DMSO-d 6 , $\left.75 \mathrm{MHz}\right) \delta: 18.4,39.8\left(2 \mathrm{CH}_{2}\right), 117.6(\mathrm{CN}), 119.0,126.9,130.2,132.0,137.8,142.4$, 143.7 (benzene, thiophene C), $193.7(\mathrm{C}=\mathrm{N}), 198.1$ (CO). EIMS m/z $296[\mathrm{M}]^{+}$(12); Analysis Calcd for $\mathrm{C}_{15} \mathrm{H}_{12} \mathrm{~N}_{4} \mathrm{OS}$ (296.35): C, 60.79; H, 4.08; N, 18.91; S, 10.82. Found: C, 60.84; H, 3.79; N, 18.69; 10.63.

Compound 22: Yellow crystals (EtOH), yield $76 \%(2.17 \mathrm{~g}), \mathrm{mp} 170^{\circ} \mathrm{C}-173^{\circ} \mathrm{C}$. IR $(\mathrm{KBr}) \mathrm{cm}^{-1}$ : 3450 - 3328, 3056, 2945, 1738, 1671, 1615. ${ }^{1} \mathrm{H}$ NMR (DMSO-d 6 , $\left.400 \mathrm{MHz}\right): \delta=2.45-2.47$ (m, 2H, $\mathrm{CH}_{2}$ ), $2.56-2.64(\mathrm{~m}$, $2 \mathrm{H}, \mathrm{CH}_{2}$ ), 4.82 (s, $2 \mathrm{H}, \mathrm{D}_{2} \mathrm{O}$ exchangeable, $\mathrm{NH}_{2}$ ), 7.40 - 7.57 (m, 5H, $\left.\mathrm{C}_{6} \mathrm{H}_{5}\right) .{ }^{13} \mathrm{C}$ NMR (DMSO- $\left.\mathrm{d}_{6}, 75 \mathrm{MHz}\right): \delta=$ 18.4, $39.2\left(2 \mathrm{CH}_{2}\right), 117.6,119.0,126.9,130.0,130.2,131.9,140.9,142.0$ (benzene, pyridazine, thiophene C), $193.7(\mathrm{C}=\mathrm{N}), 198.1(\mathrm{C}=\mathrm{O})$. EIMS m/z $282[\mathrm{M}]^{+}(20)$; Analysis Calcd for $\mathrm{C}_{15} \mathrm{H}_{11} \mathrm{~N}_{3} \mathrm{O}_{2} \mathrm{~S}$ (297.33): C, 60.59; H, 3.73; N, 14.13; S, 10.78. Found: C, 60.85; H, 3.72; N, 14.28; S, 11.09.

2,4-Diphenyl-3-thioxo-3,4,6,7-tetrahydrobenzo[e][1,2,4]triazin-8(2H)-one (23)

To a solution of compound 18a ( $2.16 \mathrm{~g}, 0.01 \mathrm{~mol})$ in 1,4-dioxane $(40 \mathrm{~mL})$ containing triethylamine $(0.50 \mathrm{~mL})$, phenylisothiocyanate $(1.30 \mathrm{~g}, 0.01 \mathrm{~mol})$ was added. The reaction mixture was heated under reflux for $4 \mathrm{~h}$ then poured onto ice/water containing few drops of hydrochloric acid and the formed solid product was collected by filtration.

Compound 23: Pale yellow crystals $(\mathrm{EtOH})$, yield $62 \%(2.06 \mathrm{~g}), \mathrm{mp} 220^{\circ} \mathrm{C}-222^{\circ} \mathrm{C}$. IR $(\mathrm{KBr}) \mathrm{cm}^{-1}$ : 3046, 2944, 1720, 1680, 1624. ${ }^{1} \mathrm{H}$ NMR (DMSO-d 6 , $\left.400 \mathrm{MHz}\right): \delta=1.85-1.92\left(\mathrm{~m}, 2 \mathrm{H}, \mathrm{CH}_{2}\right), 2.40-2.62(\mathrm{~m}, 2 \mathrm{H}$, $\mathrm{CH}_{2}$ ), $7.12\left(\mathrm{~m} \mathrm{1H}\right.$, cyclohexene CH), $7.33-7.61\left(\mathrm{~m}, 10 \mathrm{H}, 2 \mathrm{C}_{6} \mathrm{H}_{5}\right) .{ }^{13} \mathrm{C}$ NMR (DMSO-d $\left.\mathrm{d}_{6}, 75 \mathrm{MHz}\right) \delta$ : 33.0, 39.6 $\left(2 \mathrm{CH}_{2}\right), 117.5(\mathrm{CH}), 117.9,124.1,124.9,126.9,128.9,130.3,140.0$ (two benzene, pyridazine C), 193.7193 .8 $(\mathrm{C}=\mathrm{N}, \mathrm{C}=\mathrm{S} \text { ), } 198.1 \text { (CO). EIMS m/z } 333 \text { [M] }]^{+}$(28); Analysis Calcd for $\mathrm{C}_{19} \mathrm{H}_{15} \mathrm{~N}_{3} \mathrm{OS}$ (333.41): C, 68.45; H, 4.53; N, 12.60; S, 9.62. Found: C, 68.29; H, 4.71; N, 12.49; S, 9.77.

2-(Amino(3-imino-8-oxo-2-phenyl-2,3,5,6,7,8-hexahydrocinnolin-4-yl)methylene)malononitrile (24)

To a dry solid of compound 18a (2.16 g, $0.01 \mathrm{~mol})$, compound 6 (1.33 g, $0.01 \mathrm{~mol})$ and ammonium acetate ( $0.50 \mathrm{~g}$ ) were added. The reaction mixture, in each case was heated in an oil bath at $120^{\circ} \mathrm{C}$ for $15 \mathrm{~min}$. The solid product produced after boiling with ethanol was collected by filtration.

Compound 24: Yellowcrystals (EtOH), yield 80\% (2.31 g), m.p. $210^{\circ} \mathrm{C}-213^{\circ} \mathrm{C}$. IR $(\mathrm{KBr}) \mathrm{cm}^{-1}: 3545-3373$, 2934, 2220 - 2190, 1680, 1655, 1630. ${ }^{1} \mathrm{H}$ NMR (DMSO-d 6 , $400 \mathrm{MHz}$ ): $\delta=1.48-2.50$ (m, 2H, CH ), $2.60-2.68$ (m, 4H, 2 $\mathrm{CH}_{2}$ ), 3.31 (s, $2 \mathrm{H}, \mathrm{D}_{2} \mathrm{O}$ exchangeable, $\mathrm{NH}_{2}$ ), $7.48-7.62\left(\mathrm{~m}, 5 \mathrm{H}, \mathrm{C}_{6} \mathrm{H}_{5}\right), 14.70(\mathrm{~s}, 1 \mathrm{H}, \mathrm{NH}) .{ }^{13} \mathrm{C}$ NMR (DMSO-d $\left.\mathrm{d}_{6}, 75 \mathrm{MHz}\right): \delta=18.3,39.7,40.1\left(3 \mathrm{CH}_{2}\right), 118.2,118.6(2 \mathrm{CN}), 117.2,118.0(2 \mathrm{CN}), 119.2,120.6(\mathrm{C}=\mathrm{C})$, 121.7, 124.4, 129.6 132.1, 141.5, 144.6(benzene, pyridazine $\mathrm{C}), 193.7(\mathrm{C}=\mathrm{N}), 198.2,196.8$ (C=O). EIMSm/z330 $[\mathrm{M}]^{+}$(28); Analysis Calcd for $\mathrm{C}_{18} \mathrm{H}_{14} \mathrm{~N}_{6} \mathrm{O}$ (330.34): C, 65.44; H, 4.27; N, 25.44. Found: C, 65.51; H, 4.29; N, 25.63.

2,4-Diamino-7-oxo-8-(2-phenylhydrazono)-5,6,7,8-tetrahydronaphthalene-1,3-dicarbonitrile (25)

To a solution of compound $18 \mathrm{a}(2.61 \mathrm{~g}, 0.01 \mathrm{~mol})$ in 1,4-dioxane $(40 \mathrm{~mL})$ containing triethylamine $(0.50 \mathrm{~mL})$, 
compound 6 (1.33 g, $0.01 \mathrm{~mol}$ ) was added. The reaction mixture was heated under reflux for $6 \mathrm{~h}$. The solid product, formed in each case, upon pouring onto ice/water mixture containing few drops of hydrochloric acid.

Compound 25: White crystals (EtOH), yield $73 \%(2.40 \mathrm{~g}), \mathrm{mp} 212^{\circ} \mathrm{C}-215^{\circ} \mathrm{C}$. IR (KBr) $\mathrm{cm}^{-1}: 3642-3340$, 3055, 2944, 2220, 2206, 1680, 1630. ${ }^{1} \mathrm{H}$ NMR (DMSO-d 6 , $400 \mathrm{MHz}$ ): $\delta=1.82-1.96$ (m, 2H, $\mathrm{CH}_{2}$ ), $2.20-2.50$ $\left(\mathrm{m}, 2 \mathrm{H}, \mathrm{CH}_{2}\right), 3.06,3.86\left(2 \mathrm{~s}, 4 \mathrm{H}, \mathrm{D}_{2} \mathrm{O}\right.$ exchangeable, $\left.2 \mathrm{NH}_{2}\right), 7.25-7.61\left(\mathrm{~m}, 5 \mathrm{H}, \mathrm{C}_{6} \mathrm{H}_{5}\right), 10.62\left(\mathrm{~s}, 1 \mathrm{H}, \mathrm{D}_{2} \mathrm{O}\right.$ exchangeable NH). ${ }^{13} \mathrm{C}$ NMR (DMSO-d $\left.{ }_{6}, 75 \mathrm{MHz}\right): \delta=20.6,36.9\left(2 \mathrm{CH}_{2}\right), 116.8,117.3(2 \mathrm{CN}), 127.4,128.8$, 128.6, 129.0, 129.2, 129.8, 140.5 (two benzene C), $193.7(\mathrm{C}=\mathrm{N}), 196.2(\mathrm{C}=\mathrm{O})$. EIMSm/z 330 [M] ${ }^{+}$(38); Analysis Calcd for $\mathrm{C}_{18} \mathrm{H}_{14} \mathrm{~N}_{6} \mathrm{O}$ (330.34): C, 65.44; H, 4.27; N, 25.44. Found: C, 65.73; H, 4.39; N, 25.68.

\section{Conclusion}

In summary, we have shown herein that our strategy is compatible with the synthesis of a wide range of cyclohexane-1,3-dione derivatives and particularly when being corporate to heterocyclic and fused derivatives. The cytotoxicity of the newly synthesized products were evaluated against human gastric cancer (NUGC and HR), human colon cancer (DLD1), human liver cancer (HA22T and HEPG2), human breast cancer (MCF), nasopharyngeal carcinoma (HONE1) and normal fibroblast cells (WI38). The results showed that compounds $\mathbf{3 b}, \mathbf{5 c}, \mathbf{7 b}$, 10b, 12, 14b, 16, 18b, 19b, 20b, 21 and 24 exhibited optimal cytotoxic effect against cancer cell lines, with $\mathrm{IC}_{50}$ 's in the nM range. In addition, compounds $\mathbf{1 6}$ and $\mathbf{2 1}$ showed non toxicity when tested in vivo lethality toshrimp larvae (Artemiasalina).

\section{References}

[1] Sevenard, D.V., Vorobyev, M., Sosnovskikh, V.Y., Wessel, H., Kazakova, O., Vogel, V., Shevchenko, N.E., Nenajdenko, V.G., Lork, E. and Röschenthaler, G.V. (2009) Halogenation of Fluorinated Cyclic 1,3-Dicarbonyl Compounds: New Aspects of Synthesis Application. Tetrahedron, 65, 7538-7552. http://dx.doi.org/10.1016/j.tet.2009.06.122

[2] Ohtsuka, Y., Uraguchi, D., Yamamoto, K., Tokuhisa, K. and Yamakawa, T. (2012) Synthesis of 2-(Trifluouromethyl)1,3-Dicarbonyl Compounds through Direct Trifluoromethylation with $\mathrm{CF}_{3} \mathrm{I}$ and Their Application to Fluorinated Pyrazoles Syntheses. Tetrahedron, 68, 2636-2649. http://dx.doi.org/10.1016/j.tet.2012.01.075

[3] Antonioletti, R., Bovicelli, P. and Malancona, S. (2002) A New Route to 2-Alkenyl-1,3-Dicarbonyl Compounds, Intermediates in the Synthesis of Dihydrofurans. Tetrahedron, 58, 589-596. http://dx.doi.org/10.1016/S0040-4020(01)01173-5

[4] Chatterjee, P.N. and Roy, S. (2011) Alkylation of 1,3-Dicarbonyl Compounds with Benzylic and Propargylic Alcohols Using Irbimetallic Catalyst: Synthesis of Fully Decorated Furans and Pyrroles. Tetrahedron, 67, 4569-4577. http://dx.doi.org/10.1016/j.tet.2011.04.092

[5] Narayanaperumal, S., Silva, R.C., Feu, K.S., Torre, A.F., Corrêa, A.G. and Paixão, M.W. (2013) Basic-Functionalized Recyclable Ionic Liquid Catalyst: A Solvent-Free Approach for Michael Addition of 1,3-Dicarbonyl Compounds Tonitroalkenes under Ultrasound Irradiation. Ultrasonics Sonochemistry, 20, 793-798.

[6] Arcadi, A., Alfonsi, M., Chiarini, M. and Marinelli, F. (2009) Sequential Gold-Catalyzed Reactions of 1-Phenylprop2-yn-1-ol with 1,3-Dicarbonyl Compounds. Journal of Organometallic Chemistry, 694, 576-582. http://dx.doi.org/10.1016/j.jorganchem.2008.12.013

[7] Nadaraj, V., Thamarai Selvi, S.T. and Mohan, S. (2009) Microwave-Induced Synthesis and Anti-Microbial Activities of 7,10,11,12-Tetrahydrobenzo[c]acridin-8(9H)-One Derivatives. European Journal of Medicinal Chemistry, 44, 976980. http://dx.doi.org/10.1016/j.ejmech.2008.07.004

[8] Goncalves, S., Nicolas, M., Wagner, A. and Baati, R. (2010) Exploring the One-Pot C-Acylation of Cyclic 1,3-Diones with Unactivated Carboxylic Acid. Tetrahedron Letters, 51, 2348-2350. http://dx.doi.org/10.1016/j.tetlet.2010.02.111

[9] Mulongo, G., Mbabazi, J., Odongkara, B., Twinomuhwezi, H. and Mpango, G.B. (2011) New Biologically Active Compounds from 1,3-Diketones. Journal of Chemical Sciences, 1, 102-108.

[10] Schulz, A., Ort, O., Beyer, P. and Kleinig, H. (1993) SC-0051, a 2-Benzoyl-Cyclohexane-1,3-Dione Bleaching Herbicide, Is a Potent Inhibitor of the Enzyme p-Hydroxyphenylpyruvate Dioxygenase. FEBS Letters, 318, 162-166. http://dx.doi.org/10.1016/0014-5793(93)80013-K

[11] Soeda, T. and Uchida, T. (1987) Inhibition of Pigment Synthesis by 1,3-dimethyl-4-(2,4-dichlorobenzoyl)-5-hydroxypyrazole, Norflurazon, and New Herbicidal Compounds in Radish and Flatsedge Plants. Pesticide Biochemistry and Physiology, 29, 35-42.

[12] Kamel, M.M., El-Ansary, A.K. and Milad, Y.R. (2013) Design, Synthesis and Cytotoxicity of Pyridine, Pyrazole and Thiazole Derivatives Derived from N-alkyl-4,5,6,7-tetrahydro-1-benzothiophene. Chemistry of Heterocyclic Com- 
pounds, 49, 392-403. http://dx.doi.org/10.1007/s10593-013-1259-4

[13] Mohareb, R.M. and Al-Omran, F. (2012) Reaction of Pregnenolone with Cyanoacetyl-Hydrazine: Novel Synthesis of Hydrazide-Hydrazone, Pyrazole, Pyridine, Thiazole, Thiophene Derivatives and Their Cytotoxicity Evaluations. Steroids, 77, 1551-1559.

[14] Mohareb, R.M., El-Sayed, N.E. and Abdelaziz, M.A. (2013) The Knoevenagel Reactions of Pregnenolone with Cyanomethylene Reagents: Synthesis of Thiophene, thieno[2,3-b]pyridine, thieno[3,2- $d$ ] isoxazole Derivatives of Pregnenolone and Their in Vitro Cytotoxicity towards Tumor and Normal Cell Lines. Steroids, 78, 1209-1219. http://dx.doi.org/10.1016/j.steroids.2013.08.007

[15] Zhang, W., Benmohamed, R., Arvanites, A.C., Morimoto, R.I., Ferrante, R.J., Kirsch, D.R. and Silverman, R.B. (2012) Cyclohexane 1,3-diones and Their Inhibition of Mutant SOD1-Dependent Protein Aggregation and Toxicity in PC12 Cells. Bioorganic \& Medicinal Chemistry, 20, 1029-1045. http://dx.doi.org/10.1016/j.bmc.2011.11.039

[16] Zhang, Z.H., Zhang, P., Yang, S.H., Wang, H.J. and Deng, J. (2010) $\beta$-Cyclodextrin Mediated Synthesis of 1,8-Dioxooctahydroxanthenes in Water. Journal of Chemical Sciences, 122, 427-432. http://dx.doi.org/10.1007/s12039-010-0049-0

[17] Lock, E.A., Gaskin, P., Ellis, M., Provan, W.M. and Smith, L.L. (2006) Tyrosinemia Produced by 2-(2-nitro-4-trifluoromethylbenzoyl)-cyclohexane-1,3-dione (NTBC) in Experimental Animals and Its Relationship to Corneal Injury. Toxicology and Applied Pharmacology, 215, 9-16. http://dx.doi.org/10.1016/j.taap.2006.01.015

[18] Tehranchian, S., Akbarzadeh, T., Fazeli, M.R., Jamalifar, H. and Shafiee, A. (2005) Synthesis and Antibacterial Activity of 1-[1,2,4-triazol-3-yl] and 1-[1,3,4-thiadiazol-2-yl]-3-methylthio-6,7-dihydrobenzo[c]thiophen-4(5H)ones. Bioorganic \& Medicinal Chemistry Letters, 15, 1023-1025. http://dx.doi.org/10.1016/j.bmcl.2004.12.039

[19] Atta-ur-Rahman, Choudhary, M.I. and Thomsen, W.J. (2001) Bioassay Techniques for Drug Development. Harwood Academic Publishers, Newark, 9-10.

[20] Ballantyne, B., Marrs, T.C. and Syversen, T. (2009) General and Applied Toxicology. 2nd Edition, Vol. 1, 52 p.

[21] Carballo, J.L., Inda, Z.L.H., Pérez, P. and Grávalos, M.D.G. (2002) A Comparison between Two Brine Shrimp Assays to Detect in Vitro Cytotoxicity in Marine Natural Products. BMC Biotechnology, 2, 17. http://dx.doi.org/10.1186/1472-6750-2-17

[22] Calleja, M.C. and Persoone, G. (1992) The Potential of Ecotoxicological Tests for the Prediction of Acute Toxicityin Man as Evaluated on the 1st 10 Chemicals on the Meic Program. ATLA-Alternatives to Laboratory Animals, 20, $396-$ 405. 